\title{
Convection and AGN feedback in clusters of galaxies
}

\author{
Benjamin D. G. Chandran \\ benjamin. chandran@unh.edu \\ Space Science Center and Department of Physics, University of New Hampshire \\ Yann Rasera \\ yann.rasera@unh.edu \\ Space Science Center and Department of Physics, University of New Hampshire
}

\begin{abstract}
A number of studies have shown that the convective stability criterion for the intracluster medium (ICM) is very different from the Schwarzchild criterion due to the effects of anisotropic thermal conduction and cosmic rays. Building on these studies, we develop a model of the ICM in which a central active galactic nucleus (AGN) accretes hot intracluster plasma at the Bondi rate and produces cosmic rays that cause the ICM to become convectively unstable. The resulting convection heats the intracluster plasma and regulates its temperature and density profiles. By adjusting a single parameter in the model (the size of the cosmic-ray acceleration region), we are able to achieve a good match to the observed density and temperature profiles in a sample of eight clusters. Our results suggest that convection is an important process in cluster cores. An interesting feature of our solutions is that the cooling rate is more sharply peaked about the cluster center than is the convective heating rate. As a result, in several of the clusters in our sample, a compact cooling flow arises in the central region with a size $r_{\text {cf }}$ that is typically a few kpc. The cooling flow matches onto a Bondi flow at smaller radii. The mass accretion rate in the Bondi flow is equal to, and controlled by, the rate at which mass flows in through the cooling flow. Our solutions suggest that the AGN regulates the mass accretion rate in these clusters by controlling $r_{\mathrm{cf}}$ : if the AGN power rises above the equilibrium level, $r_{\mathrm{cf}}$ decreases, the mass accretion rate drops, and the AGN power drops back down to the equilibrium level.
\end{abstract}

Subject headings: cooling flows — galaxies:clusters:general — galaxies:active — convection - magnetic fields — turbulence 


\section{Introduction}

Active galactic nuclei (AGNs) have enormous mechanical and radiative luminosities. If an AGN's power can be transferred to the surrounding interstellar and intergalactic media, the resulting heating can have a large effect on the ambient plasma. There has recently been great interest in this process of "AGN feedback," its role in galaxy formation, and the possibility that AGN feedback solves the over-cooling problem (Suginohara \& Ostriker 1998, Lewis et al 2000, Tornatore et al 2003, Nagai \& Kravtsov 2004) and cooling-flow problem for clusters of galaxies (Böhringer et al 20001; David et al 2001; Tamura et al 2001; Molendi \& Pizzolato 2001; Blanton, Sarazin, \& McNamara 2003; Peterson et al 2001, 2003).

One of the main unsolved problems for AGN feedback is to understand how AGN power is transferred to the diffuse ambient plasma. A number of mechanisms have been investigated, including Compton heating (Binney \& Tabor 1995; Ciotti \& Ostriker 1997, 2001; Ciotti, Ostriker, \& Pellegrini 2004, Sazonov et al 2005), shocks (Tabor \& Binney 1993, Binney \& Tabor 1995), magnetohydrodynamic (MHD) wave-mediated plasma heating by cosmic rays (Böhringer \& Mor-

fill 1988; Rosner \& Tucker 1989; Loewenstein, Zweibel, \& Begelman 1991), and cosmic-ray bubbles produced by the central AGN (Churazov et al 2001, 2002; Reynolds 2002; Brüggen 2003; Reynolds et al 2005), which can heat intracluster plasma by generating turbulence (Loewenstein \& Fabian 1990, Churazov et al 2004) and sound waves (Fabian et al 2003; Ruszkowski, Brüggen, \& Begelman 2004a,b) and by doing $p d V$ work (Begelman 2001, 2002; Ruszkowski \& Begelman 2002; Hoeft \& Brüggen 2004). Despite this substantial progress, it is still not clear how AGN feedback controls the density and temperature profiles of the ambient plasma in a way that is both self-regulating and consistent with observations.

In this paper, we focus on clusters of galaxies and explore the hypothesis that central AGNs heat and regulate the intracluster plasma by causing the intracluster medium to become convectively unstable, a scenario that was investigated in two earlier studies [Chandran (2004) (hereafter Paper I) and Chandran (2005) (hereafter Paper II)]. At first glance, this hypothesis seems obviously incorrect, since observations show that the specific entropy $s$ in intracluster plasmas increases with radius $r$. However, several recent studies have shown that the Schwarzchild criterion $(d s / d r>0)$ does not apply to low-density, magnetized plasmas such as those found in clusters, in which the charged-particle gyroradii are much less than the Coulomb mean free path. In such plasmas, heat and charged particles diffuse primarily along magnetic field lines, and only weakly across the magnetic field. This anisotropy turns out to have a profound effect on convective stability, as shown analytically by Balbus $(2000,2001)$ and numerically by Parrish \& Stone $(2005,2007)$. These authors considered a stratified plasma in which the gravitational acceleration is in the $-z$ direction and the equilibrium magnetic field is in the $x y$-plane and showed that the convective stability criterion is $d T / d z>0$, not $d s / d z>0$, where $T$ is the temperature. When cosmic rays are present, the con- 
vective stability criterion becomes $n k_{B} d T / d z+d p_{\text {cr }} / d z>0$, as shown analytically by Chandran $\&$ Dennis (2006) and numerically by Rasera $\&$ Chandran (2007). Here, $n$ and $p_{\mathrm{cr}}$ are the thermalplasma number density and cosmic-ray pressure, respectively. In galaxy clusters, the gravitational acceleration is in the $-r$ direction, and the convective stability criterion is

$$
n k_{B} \frac{d T}{d r}+\frac{d p_{\mathrm{cr}}}{d r}>0 .
$$

(Paper II and appendix B provide a more extensive discussion.) Although $d T / d r>0$ in cluster cores, equation (11) shows that cosmic rays produced by an AGN at the center of a cluster can lead to convective instability, since centrally produced cosmic rays satisfy $d p_{\mathrm{cr}} / d r<0$.

In this paper, we construct a spherically symmetric, steady-state model of convective intracluster plasmas using mixing-length theory, and compare this model to observations. We assume that a central supermassive black hole accretes hot intracluster plasma at the Bondi rate, and converts a small fraction of the accreted rest-mass energy into cosmic rays that are accelerated by shocks within some distance $r_{\text {source }}$ of the center of the cluster. The resulting cosmic-ray pressure gradient leads to convection, which in turn heats the thermal plasma in the cluster core by advecting internal energy inwards and allowing the cosmic rays to do $p d V$ work on the thermal plasma. The model also includes thermal conduction, cosmic-ray diffusion, and radiative cooling. The model involves much less emission from plasma at temperatures below one-third of the cluster's average temperature than the cooling flow model (Fabian 1994), and thus offers a possible solution to the cooling-flow problem.

We compare the density and temperature profiles predicted by the model to the profiles inferred from X-ray observations of eight clusters. We adjust a single parameter, the size $r_{\text {source }}$ of the cosmic-ray acceleration region, to optimize the fit. The model solutions match the observations well, with the exception of the density with the central $\simeq 50 \mathrm{kpc}$ of Sersic 159-03, which is underestimated by the model. We suggest a possible explanation for this discrepancy in section 3 , We also find that the cosmic-ray luminosities of the AGN in our sample are strongly correlated with the observationally inferred mechanical luminosities of these AGN. Our results suggest that AGN-driven convection is an important process in cluster cores.

An attractive feature of this model and other models based on AGN feedback and Bondi accretion is that they are self-regulating. One argument for why Bondi accretion is self-regulating was advanced by Nulsen (2004) and Böhringer et al (2004), who noted that the Bondi accretion rate is a monotonically decreasing function of the specific entropy near the center of the cluster. Thus, if the central plasma becomes too cool, the Bondi accretion rate rises, the AGN feedback heating increases, and the specific entropy of the central plasma rises back to its equilibrium value. In this paper, we offer an additional explanation for how AGN heating on large scales ( $\gtrsim 5 \mathrm{kpc}$ ) can regulate the mass accretion rate onto the central black hole. In our solutions, we find that the 
radiative cooling rate is more sharply peaked about the center of a cluster than is the convective heating rate. As a result, in several of the clusters in our sample, the central region becomes a cooling flow. The radius of this cooling flow, $r_{\mathrm{cf}}$, is typically a few kpc in our solutions. At smaller radii, the flow makes a transition from a cooling flow to a Bondi flow. However, as in the work of Quataert \& Narayan (2000), the mass accretion rate of the inner Bondi flow is controlled by the surrounding cooling flow. In our model, which has no mass dropout, the mass accretion rate is approximately the plasma mass interior to $r_{\mathrm{cf}}$ divided by the cooling time at $r_{\mathrm{cf}}$. The AGN then regulates the mass accretion rate by controlling $r_{\mathrm{cf}}$ : if the AGN power rises above the equilibrium level, the size of the central cooling flow decreases, the mass accretion rate drops, and the AGN power then drops back down to the equilibrium level.

This paper extends the previous models of paper I and paper II in several ways. In contrast to paper I, the present paper takes into account the role of anisotropic thermal conduction and cosmicray diffusion, which strongly modify the convective stability criterion. In contrast to paper II, we take the cosmic-ray acceleration to occur within a relatively small fraction of the total volume at any given radius, which allows for localized pockets of excess cosmic-ray pressure that tend to rise buoyantly. We also take into account the nonzero average radial velocity, and compare the model to a larger sample of clusters.

The rest of this paper is organized as follows. We present the basic equations of the model in section 2, In section 3, we compare our model calculations to observations. In section 4 we consider the radial profiles of the different heating rates and the factors that determine whether AGN feedback or thermal conduction is the dominant heat source at $r \lesssim 100 \mathrm{kpc}$. In section 5 we discuss the central cooling flows that arise in our model solutions for several of the clusters in our sample. We also comment in section 5 on the viability of the Bondi accretion model for the AGN at the centers of clusters. We summarize our results in section 6 , We present results on the radial profiles of the turbulent velocity and cosmic-ray pressure in appendix $\mathrm{A}$. In appendix $\mathrm{B}$ we present a systematic derivation of the two-fluid mixing-length theory that we employ in our model.

\section{Model equations}

We describe the intracluster medium using a standard set of two-fluid equations for cosmic rays and thermal plasma (Drury \& Volk 1981, Jones \& Kang 1990), modified to include thermal conduction, viscous dissipation, and radiative cooling:

$$
\begin{gathered}
\frac{d \rho}{d t}=-\rho \nabla \cdot v \\
\rho \frac{d v}{d t}=-\nabla\left(p+p_{\mathrm{cr}}\right)-\rho \nabla \Phi-\nabla \cdot \Pi_{\mathrm{visc}}
\end{gathered}
$$




$$
\frac{d p}{d t}=-\gamma p \nabla \cdot v+(\gamma-1)\left[H_{\mathrm{visc}}+\nabla \cdot(\kappa \cdot \nabla T)-R\right],
$$

and

$$
\frac{d p_{\mathrm{cr}}}{d t}=-\gamma_{\mathrm{cr}} p_{\mathrm{cr}} \nabla \cdot v+\nabla \cdot\left(\mathrm{D} \cdot \nabla p_{\mathrm{cr}}\right)+\left(\gamma_{\mathrm{cr}}-1\right) \dot{E}_{\mathrm{source}}
$$

where

$$
\frac{d}{d t} \equiv \frac{\partial}{\partial t}+v \cdot \nabla,
$$

$\rho$ is the plasma density, $v$ is the bulk velocity of the two-fluid mixture, $p$ and $p_{\mathrm{cr}}$ are the plasma and cosmic-ray pressures, $T$ is the plasma temperature, $\Phi$ is the gravitational potential, $\Pi_{\text {visc }}$ is the viscous stress tensor, $\gamma$ and $\gamma_{\mathrm{cr}}$ are the plasma and cosmic-ray adiabatic indices (which are treated as constants), $H_{\mathrm{visc}}$ is the rate of viscous heating, $\mathrm{K}$ is the thermal conductivity tensor, $R$ is the radiative cooling rate, $\dot{E}_{\text {source }}$ is the rate of injection of cosmic-ray energy per unit volume by the central radio source, and $\mathrm{D}$ is an effective momentum-averaged cosmic-ray diffusion tensor. For the calculations presented in section 3, we set $\gamma=5 / 3$ and $\gamma_{\mathrm{cr}}=4 / 3$. We ignore radiative cooling of cosmic rays, which is reasonable if protons make the dominant contribution to the cosmic-ray pressure. We also neglect Coulomb interactions between cosmic rays and thermal plasma, as well as wave-mediated heating of the thermal plasma by cosmic rays. As discussed below, we take into account the effects of the magnetic field on $\kappa$ and $D$, but we neglect the Lorentz force and resistive dissipation. In the following subsections, we describe the approximations we use to solve the above equations.

\subsection{Mixing length theory}

To account for convection, we write each fluid quantity as an average value plus a turbulent fluctuation:

$$
\begin{aligned}
& v=\langle v\rangle+\delta v, \\
& p=\langle p\rangle+\delta p,
\end{aligned}
$$

etc, where $\langle\ldots\rangle$ denotes an average over the turbulent fluctuations. We take the averaged quantities to be spherically symmetric and independent of time, and we treat the fluctuating quantities as small. To obtain equations for the average cluster properties, we average equations (2) through (5). We evaluate the averages $\langle\rho v\rangle,\langle v p\rangle$, and $\left\langle v p_{\text {cr }}\right\rangle$ in equations (2), (4), and (5) using a two-fluid mixing-length theory that we describe in appendix B. The essential idea behind this theory is that the amplitudes of the turbulent fluctuations increase as the average plasma and cosmic-ray profiles move past the point of marginal stability towards increasing degrees of convective instability. As this happens, the magnitudes of the internal energy flux $\langle v p\rangle /(\gamma-1)$ and the cosmic-ray energy flux $\left\langle v p_{\mathrm{cr}}\right\rangle /\left(\gamma_{\mathrm{cr}}-1\right)$ increase, which in turn affects the density, temperature, and cosmic-ray-pressure 
profiles. The two-fluid mixing length theory provides an approximate way of determining the resulting profiles as well as the $r$-dependent turbulent velocity in a self-consistent way. A key parameter of the model is the mixing length $l$, which characterizes the length scale of the convective turbulence. We set

$$
l=0.4 r
$$

\subsection{Hydrostatic equilibrium}

We assume that the convection is subsonic and confine our model to $r \geq 0.2 \mathrm{kpc}$, so that the average radial velocity remains subsonic throughout our solutions. As a result, we can to a reasonable approximation drop the inertial terms in the average of equation (3). The viscous term in equation (3) is important primarily for dissipating small-scale velocity fluctuations and can also be neglected in the average of equation (3). The average of equation (3) then reduces to

$$
\frac{d}{d r}\left\langle p_{\text {tot }}\right\rangle=-\langle\rho\rangle \frac{d \Phi}{d r}
$$

where

$$
p_{\mathrm{tot}}=p+p_{\mathrm{cr}}
$$

\subsection{Gravitational potential}

We take the gravitational potential to be the sum of four components,

$$
\Phi=\Phi_{\mathrm{c}}+\Phi_{\mathrm{s}}+\Phi_{\mathrm{bh}}+\Phi_{\mathrm{p}}
$$

where $\Phi_{c}$ is the contribution from the the cluster's dark matter, $\Phi_{\mathrm{s}}$ is the contribution from the stars in the brightest cluster galaxy (BCG), $\Phi_{\mathrm{bh}}$ is the contribution from the black hole at $r=0$, and $\Phi_{\mathrm{p}}$ is the contribution from the intracluster plasma. We take the cluster dark matter to have an NFW density profile (Navarro, Frenk, \& White 1997),

$$
\rho_{\mathrm{DM}}=\frac{\delta_{c} \rho_{\mathrm{crit}}(z) r_{s}^{3}}{r\left(r+r_{s}\right)^{2}}
$$

where

$$
\delta_{c}=\frac{200}{3} \frac{c^{3}}{[\ln (1+c)-c /(1+c)]},
$$

$r_{s}$ is the scale radius, $c$ is the concentration parameter, and $\rho_{\text {crit }}=3 H^{2} / 8 \pi G$ is the critical density at the redshift $z$ of the cluster. The latter is calculated assuming $\Omega_{0}=0.3, \Omega_{\Lambda, 0}=0.7$, and $H_{0}=$ 
Table 1: Parameters used in determining the gravitational potential

\begin{tabular}{|c|c|c|c|c|c|c|c|c|c|}
\hline Cluster & BCG & $\begin{array}{c}r_{s} \\
(\mathrm{kpc})\end{array}$ & $c$ & $z$ & $M_{B}$ & $B-V$ & $\begin{array}{c}L_{B} \\
\left(10^{11} L_{B, \odot}\right)\end{array}$ & $\begin{array}{c}R_{e} \\
(\mathrm{kpc})\end{array}$ & $\begin{array}{c}M_{\mathrm{bh}} \\
\left(10^{9} M_{\odot}\right)\end{array}$ \\
\hline Virgo & NGC 4486 (M87) & 560 & 2.8 & (see below) & -21.96 & 0.93 & 0.938 & 5.03 & 1.38 \\
\hline Abell 262 & NGC 0708 & 85 & 8.62 & 0.0155 & -21.08 & 1.06 & 0.417 & 25.6 & 0.555 \\
\hline Sersic $159-03$ & ESO 291-009 & 159 & 6.56 & 0.0572 & -22.16 & 1.00 & 1.13 & 29.5 & 1.92 \\
\hline Abell 4059 & ESO 349-010 & 744 & 2.7 & 0.0466 & -22.73 & 1.06 & 1.91 & 24.5 & 4.12 \\
\hline Hydra A & PGC 026269 & 77 & 12.3 & 0.0550 & -22.97 & 0.82 & 2.38 & 39.6 & 4.12 \\
\hline Abell 496 & PGC 015524 & 129 & 7.75 & 0.0322 & -22.48 & 1.12 & 1.51 & 49.9 & 3.27 \\
\hline Abell 1795 & PGC 049005 & 430 & 4.21 & 0.0639 & -22.04 & 1.00 & 1.01 & 40.3 & 1.66 \\
\hline Perseus & NGC 1275 & 481 & 4.09 & 0.0179 & -22.62 & 0.53 & 1.72 & 15.3 & 1.89 \\
\hline
\end{tabular}

The NFW parameters $r_{s}$ and $c$ describe the clusters' dark matter density profiles. For Virgo $r_{s}$ and $c$ are taken from McLaughlin (1999). For Hydra A, $r_{s}$ and $c$ are taken from David et al (2001). For all other clusters, $r_{s}$ and $c$ are taken from table 1 of Piffaretti et al (2005). Redshifts $z$ are taken from Kaastra et al (2004), except for Virgo - Kaastra et al (2004) take the distance to Virgo to be $16 \mathrm{Mpc}$, and we use the same value. Absolute B-band magnitudes $M_{B}$ and $B-V$ color indices for the brightest cluster galaxies (BCGs) are taken from the "Hyperleda" database of Paturel et al (2003). The BCG effective radii $R_{e}$ are taken from Schombert (1987) for Perseus and Abell 1795, from Graham et al (1996) for Hydra A, Abell 262, and Abell 496, and from "Hyperleda" for Virgo, Sersic 159-03 and Abell 4059. $L_{B}$ is the BCG B-band luminosity. The black hole masses are determined using the mass-luminosity relation given in equation (6) of Lauer et al (2007).

$70 \mathrm{~km} \mathrm{~s}^{-1} \mathrm{Mpc}^{-1}$. The values of $r_{s}, c$, and $z$ for the eight clusters we consider in section 3 are taken from the literature and listed in table 1 .

We take the stellar mass density to have a Hernquist profile in which the stellar mass interior to radius $r$ is

$$
M_{\text {stars }}(r)=\frac{M_{0} r^{2}}{(r+a)^{2}},
$$

where $M_{0}$ is the total stellar mass and $a$ is a scale length equal to $R_{e} / 1.8153$, where $R_{e}$ is the radius of the isophote enclosing half the galaxy's light. (Hernquist 1990) As in Graham et al (2006), we set $M_{0}=\Upsilon_{B} L_{B}$, where $L_{B}$ is the BCG B-band luminosity, and $\Upsilon_{B}=5.3 M_{\odot} / L_{B, \odot}$ is the B-band stellar mass-to-light ratio for a 12-Gyr-old single stellar population (Worthey 1994). We set $L_{B} / L_{B, \odot}=10^{0.4\left(M_{B, \odot}-M_{B}\right)}$, where $M_{B, \odot}$ and $M_{B}$ are, respectively, the solar and BCG absolute B-band magnitudes, and $M_{B, \odot}=5.47$ (Cox 2000). The values of $R_{e}$ and $M_{B}$ for each cluster are taken from the literature (see table 1 ). 
We determine the black-hole mass using the mass-luminosity relation given in equation (6) of Lauer et al (2007):

$$
\log \left(\frac{M_{\mathrm{bh}}}{M_{\odot}}\right)=8.67-0.528\left(M_{V}+22\right),
$$

where $M_{V}$ is the BCG absolute V-band magnitude. We set $M_{V}=M_{B}-(B-V)$, where $M_{B}$ and the $B-V$ color index for each cluster are taken from the "Hyperleda" database (Paturel et al 2003) and listed in table 1. The resulting values of $M_{\mathrm{bh}}$ for each cluster are also listed in table 1 .

The contribution to the gravitational potential from the intracluster plasma $\Phi_{\mathrm{p}}$ is not determined ahead of time, but is instead obtained by solving $\nabla^{2} \Phi_{p}=-4 \pi G\langle\rho\rangle$, where $\langle\rho\rangle$ is the average plasma density that results from solving the model equations.

\subsection{Radiative cooling and chemical composition}

We use the analytic fit of Tozzi \& Norman (2001) to approximate the full cooling function for free-free and line emission:

$$
R=n_{\mathrm{i}} n_{\mathrm{e}}\left[0.0086\left(\frac{k_{\mathrm{B}} T}{1 \mathrm{keV}}\right)^{-1.7}+0.058\left(\frac{k_{\mathrm{B}} T}{1 \mathrm{keV}}\right)^{0.5}+0.063\right] \cdot 10^{-22} \operatorname{ergs~cm}^{3} \mathrm{~s}^{-1},
$$

where $n_{\mathrm{i}}$ is the ion density, $n_{\mathrm{e}}$ is the electron density, $k_{\mathrm{B}}$ is the Boltzmann constant, and the numerical constants correspond to $30 \%$ solar metallicity. Because we treat the turbulent fluctuations as small, we can replace $n_{\mathrm{e}}, n_{\mathrm{i}}$, and $T$ in equation (17) by their average values when calculating $\langle R\rangle$. We take the intracluster plasma to be fully ionized and to have a uniform chemical composition, with a hydrogen mass fraction of $X=0.7$ and a helium mass fraction $Y=0.29$. We take the metals to have a mean charge to mass ratio equal to that of helium. The mean molecular weight is then

$$
\mu \equiv \frac{\rho}{\left(n_{\mathrm{e}}+n_{\mathrm{i}}\right) m_{H}}=0.62
$$

The mean molecular weight per electron is then

$$
\mu_{\mathrm{e}} \equiv \frac{\rho}{n_{\mathrm{e}} m_{H}}=1.18
$$

In addition,

$$
\frac{n_{\mathrm{i}}}{n_{\mathrm{e}}}=0.91
$$

and

$$
\frac{n_{\mathrm{e}}}{n_{\mathrm{H}}}=1.21,
$$

where $n_{\mathrm{H}}$ is the hydrogen number density. 


\subsection{Transport}

Cluster magnetic fields are easily strong enough to cause cosmic rays and heat to diffuse primarily along magnetic field lines, so that

$$
\kappa \simeq \kappa_{\|} \hat{b} \hat{b}
$$

and

$$
\mathrm{D} \simeq D_{\|} \hat{b} \hat{b}
$$

where $\hat{b}$ is the magnetic field unit vector, and $\kappa_{\|}$and $D_{\|}$are the parallel conductivity and diffusivity. We take the parallel conductivity to be the classical Spitzer thermal conductivity (Spitzer \& Harm 1953, Braginskii 1965),

$$
\kappa_{\|}=\kappa_{\mathrm{S}}=9.2 \times 10^{30} n_{\mathrm{e}} k_{\mathrm{B}}\left(\frac{k_{\mathrm{B}} T}{5 \mathrm{keV}}\right)^{5 / 2}\left(\frac{10^{-2} \mathrm{~cm}^{-3}}{n_{\mathrm{e}}}\right)\left(\frac{37}{\ln \Lambda_{\mathrm{c}}}\right) \frac{\mathrm{cm}^{2}}{\mathrm{~s}},
$$

where $\ln \Lambda_{c}$ is the Coulomb logarithm. The local anisotropy of $\kappa$ and $D$ turns out to be critical for convective stability, as discussed by Balbus (2000,2001), Parrish \& Stone (2005,2007), Chandran \& Dennis (2006), and Rasera \& Chandran (2007), and we take this anisotropy into account in our mixing length theory for intracluster convection. [See, e.g., the discussion preceding equation (B29).] However, when we average equations (2) through (5) and solve for the structure of the ICM, we are interested in the transport of heat and cosmic rays over distances much greater than the correlation length of the magnetic field, $l_{B}$, which is $\sim 1-10 \mathrm{kpc}$ (Kronberg 1994; Taylor et al 2001, 2002; Vogt \& Ensslin 2003, 2005 - see Schekochihin et al 2006 and Schekochihin \& Cowley 2006 for a recent discussion of intracluster magnetic fields and turbulence). For transport over such large scales, averaging over the turbulent magnetic field leads to an effectively isotropic conductivity, which we denote $\kappa_{T}$, that is reduced relative to $\kappa_{\|}$(Rechester \& Rosenbluth 1978, Chandran \& Cowley 1998). Theoretical studies find that the reduction is by a factor of $\sim 5-10$ (Narayan \& Medvedev 2001, Chandran \& Maron 2004, Maron, Chandran, \& Blackman 2004). In this paper, we assume that

$$
\kappa_{T}=\frac{\kappa_{\|}}{8} .
$$

We take the average of the conductive heating term to be given by

$$
\langle\nabla \cdot(\kappa \cdot \nabla T)\rangle=\frac{1}{r^{2}} \frac{d}{d r}\left[r^{2} \kappa_{T} \frac{d}{d r}\langle T\rangle\right],
$$

with $T$ set equal to $\langle T\rangle$ in equation (24). Similarly, we assume that

$$
\left\langle\nabla \cdot\left(\mathrm{D} \cdot \nabla p_{\mathrm{cr}}\right)\right\rangle=\frac{1}{r^{2}} \frac{d}{d r}\left[r^{2} D_{\mathrm{cr}} \frac{d}{d r}\left\langle p_{\mathrm{cr}}\right\rangle\right] .
$$


We take the value of $D_{\text {cr }}$ to be

$$
D_{\mathrm{cr}}=\sqrt{D_{0}^{2}+v_{d}^{2} r^{2}}
$$

where $D_{0}=10^{28} \mathrm{~cm}^{2} / \mathrm{s}$ and $v_{d}=10 \mathrm{~km} / \mathrm{s}$. The $v_{d}$ term is loosely motivated by a simplified picture of cosmic-ray "self-confinement," in which cosmic rays are scattered by waves generated by the streaming of cosmic rays along field lines. If, contrary to fact, the field lines were purely radial, efficient self-confinement would limit the average radial velocity of the cosmic rays to the Alfvén speed $v_{\mathrm{A}}$, allowing the cosmic rays to travel a distance $r$ in a time $\sim r / v_{\mathrm{A}}$. For constant $v_{\mathrm{A}}$, this scaling can be approximately recovered by taking the cosmic rays to diffuse isotropically with $D_{\text {cr }} \propto r$, the scaling that arises from equation (28) when $v_{d} r \gg D_{0}$. This self-confinement scenario is too simplistic, since in clusters field lines are tangled, $v_{\mathrm{A}}$ varies in space, and it is not known whether cosmic rays are primarily scattered by cosmic-ray-generated waves or by magnetohydrodynamic (MHD) turbulence excited by large-scale stirring of the intracluster plasma. It is not clear, however, how to improve upon equation (28). Self-confinement in the presence of tangled field lines is not well understood, and the standard theoretical treatment of scattering by MHD turbulence, which takes the fluctuations to have wave vectors directed along the background magnetic field, is known to be inaccurate (Bieber et al 1994, Chandran 2000, Yan \& Lazarian 2004). A more definitive treatment must thus await further progress in our understanding of MHD turbulence and cosmic-ray transport. The value of $D_{\|}$is needed in the mixing length theory developed below. We assume that $D_{\mathrm{cr}} / D_{\|}=\kappa_{T} / \kappa_{\|}$, and thus set

$$
D_{\|}=8 D_{\mathrm{cr}}
$$

\subsection{The mass accretion rate and cosmic-ray luminosity of the central AGN}

We assume that the black hole at $r=0$ in our model, with a mass $M_{\mathrm{BH}}$ given by equation (16), accretes intracluster plasma at the Bondi (1952) rate,

$$
\dot{M}=\frac{\pi G^{2} M_{\mathrm{BH}}^{2} \rho}{c_{\mathrm{s}}^{3}},
$$

where $c_{\mathrm{s}}$ is the adiabatic sound speed, and $\rho$ and $c_{\mathrm{s}}$ are evaluated using the average plasma parameters at the radius $r_{1}=0.2 \mathrm{kpc}$, which defines the inner boundary of our model solutions. We assume that this accretion powers a jet that leads to shocks, which in turn accelerate cosmic rays. We take the cosmic-ray luminosity to be

$$
L_{\mathrm{cr}}=\eta \dot{M} c^{2}
$$

where

$$
\eta=5 \times 10^{-3}
$$


An argument against Bondi accretion in clusters is that the radiative luminosities of AGNs in elliptical galaxies are typically several orders of magnitude smaller than the nominal Bondi accretion power, given by $P_{\mathrm{Bondi}}=0.1 \dot{M}_{\mathrm{Bondi}} c^{2}$, where $\dot{M}_{\mathrm{Bondi}}$ is the Bondi accretion rate given in equation (30). (Allen et al 2006) However, the mechanical luminosities $L_{\text {mech }}$ of these AGN are often much larger than their radiative luminosities. Moreover, in a recent study of nine AGNs in nearby X-ray luminous elliptical galaxies, Allen et al (2006) found a strong correlation between $P_{\text {Bondi }}$ (as calculated from the observed plasma temperature and density profiles) and $L_{\text {mech }}$ (as inferred from the energies and time scales required to inflate the observed x-ray cavities). Allen et al (2006) found that $L_{\text {mech }}$ can be related to $P_{\text {Bondi }}$ by a power-law fit of the form $\log \left(P_{\text {Bondi }} / 10^{43} \mathrm{erg} \mathrm{s}^{-1}\right)=$ $c_{1}+c_{2} \log \left(L_{\text {mech }} / 10^{43} \mathrm{erg} \mathrm{s}^{-1}\right)$, with $c_{1}=0.65 \pm 0.16$ and $c_{2}=0.77 \pm 0.20$, and that the fraction of $\dot{M} c^{2}$ that is converted into mechanical luminosity ranges from $1.3 \%$ for a jet power of $10^{42} \mathrm{erg} / \mathrm{s}$ to $3.7 \%$ for a jet power of $10^{44} \mathrm{erg} / \mathrm{s}$. Results consistent with these were also found by Tan $\&$ Blackman (2005). These authors reviewed studies of M87 and estimated that $L_{\text {mech }}$ is about an order of magnitude larger than the radiative luminosity, and that $L_{\text {mech }} \sim 0.01 \dot{M}_{\text {Bondi }} c^{2}$. Our choice of $\eta=0.005$ is smaller than the accretion efficiencies found in these studies, in part to provide a more conservative estimate, and in part because only part of the mechanical energy is converted into cosmic rays.

We note that Bondi accretion in clusters has been considered previously by a number of authors (e.g., Quataert \& Narayan 2000, Di Matteo et al 2002, Nulsen 2004, Böhringer et al 2004, Springel et al 2005, Cattaneo \& Teyssier 2007). Also, in Tan \& Blackman's (2005) analysis, part of the reason for the small value of $\eta$ is that part of the mass flowing in through the Bondi radius never reaches the central black hole because it forms stars in a gravitationally unstable disk. Thus, the Bondi accretion rate in our model may be significantly higher than the time derivative of the mass of the central black hole.

Pizzolato \& Soker (2005) and Soker (2006) considered a different "cold feedback" scenario for mass accretion, in which cold gas fuels the central AGN. In section 5 we address several issues related to the question of whether one expects Bondi accretion or some form of cold feedback in clusters.

\subsection{Cosmic-ray acceleration by the central radio source}

The spatial distribution of cosmic-ray injection into the ICM is not precisely known. Some clues are provided by radio observations, which show that cluster-center radio sources (CCRS) differ morphologically from radio sources in other environments. As discussed by Eilek (2004), roughly half of the CCRS in a sample of 250 sources studied by Owen \& Ledlow (1997) are "amorphous," or quasi-isotropic, presumably due to jet disruption by the comparatively high-pressure, 
high-density cluster-core plasma. With the exception of Hydra A, the CCRS in the Owen-Ledlow (1997) study are smaller than non-cluster-center sources, with most extending less than $50 \mathrm{kpc}$ from the center of the host cluster (Eilek 2004). Given these findings, we take the cosmic-ray acceleration to be concentrated within the cluster core.

In paper II, it was assumed that the cosmic rays are accelerated in an approximately volumefilling manner. In contrast, in this paper, it is assumed that cosmic-ray energy is injected into the intracluster medium in only a fraction of the volume at any given radius. We then take

$$
\dot{E}_{\text {source }}=\left\langle\dot{E}_{\text {source }}\right\rangle+\delta \dot{E}_{\text {source }}
$$

where

$$
\left\langle\dot{E}_{\text {source }}\right\rangle=S_{0} e^{-r^{2} / r_{\text {source }}^{2}}
$$

can be thought of as an average of $\dot{E}_{\text {source }}$ over spherical polar angles. The constant $r_{\text {source }}$ is a free parameter that characterizes the size of the cosmic-ray acceleration region. The constant $S_{0}$ is determined on energy grounds from the equation $L_{\mathrm{cr}}=4 \pi \int_{0}^{\infty} d r r^{2}\left\langle\dot{E}_{\text {source }}(r)\right\rangle$ and equation (31). After determining $\left\langle\dot{E}_{\text {source }}(r)\right\rangle$, we set

$$
\delta \dot{E}_{\mathrm{rms}}=\eta_{2}\left\langle\dot{E}_{\text {source }}\right\rangle
$$

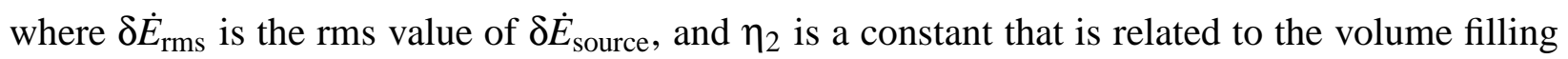
factor of the cosmic-ray acceleration region. For example, suppose that $\dot{E}_{\text {source }}=C=$ constant in a fraction $f_{\text {cr }}$ of the volume between radius $r$ and $r+d r$, and that $\dot{E}_{\text {source }}=0$ in the remainder of the volume between $r$ and $r+d r$. In this case, $\left\langle\dot{E}_{\text {source }}(r)\right\rangle=f_{\text {cr }} C,\left\langle\left[\dot{E}_{\text {source }}\right]^{2}\right\rangle=f_{\text {cr }} C^{2}$, and $\delta \dot{E}_{\mathrm{rms}}=\sqrt{\left\langle\left[\dot{E}_{\text {source }}(r)-\left\langle\dot{E}_{\text {source }}(r)\right\rangle\right]^{2}\right\rangle}=\left\langle\dot{E}_{\text {source }}(r)\right\rangle \sqrt{f_{\mathrm{cr}}^{-1}-1}$. For the calculations presented below, we set $\eta_{2}=2.5$, which corresponds to $f_{\text {cr }}=0.138$. These fluctuations in the cosmic-ray source term drive fluctuations in the fluid quantities and contribute to convection. This effect is incorporated into the two-fluid mixing length theory presented in appendix $\mathbb{B}$. The fluctuations in $\dot{E}_{\text {source }}$ result in larger fluctuations (spatial variations) in $p_{\text {cr }}$ and $\rho$ than in the model of paper II, which in some sense represent the "cosmic-ray bubbles" or X-ray cavities seen in about one-fourth of the clusters in the Chandra archive (Birzan et al 2004).

\subsection{Summary and numerical method}

The approximations described above lead to a set of coupled ordinary differential equations for the average density, temperature, and cosmic-ray pressure and the rms turbulent velocity. These equations are presented in appendix $B$. We solve this set of equations using a shooting method, in which we guess the electron density, temperature, and cosmic-ray pressure at the inner radius of 
our model $\left(r_{1}=0.2 \mathrm{kpc}\right)$ and then update these guesses until the model solution satisfies the three boundary conditions at the outer radius $r_{\text {outer }}$. These outer boundary conditions are the observed electron density $n_{\mathrm{e}, \text { outer }}$ and temperature $T_{\text {outer }}$ at $r_{\text {outer }}$, and a condition on $\left\langle d p_{\mathrm{cr}} / d r\right\rangle$ at $r_{\text {outer }}$, which amounts to requiring that $\left\langle p_{\mathrm{cr}}\right\rangle \rightarrow 0$ as $r \rightarrow \infty$. The value of $r_{\text {outer }}$ for a cluster is taken to be the radius of the first observational data point outside the cluster's cooling radius, $r_{\text {cool }}$, given in table 2 (except for Virgo, for which we take $r_{\text {outer }}$ to be the outermost data point, which lies inside of $r_{\text {cool }}$.) The values of $r_{\text {outer }}, n_{\text {outer }}$, and $T_{\text {outer }}$ are listed in table 3. After finding the values of $n_{e}, T$, and $p_{\mathrm{cr}}$ at $r_{1}$ needed to match the boundary conditions at $r_{\text {outer }}$, we integrate the equations out to radii greater than $r_{\text {outer }}$ as needed to compare to the data. A more extensive discussion of our numerical method is given in appendix $\mathrm{B}$.

\section{Comparison to observations}

We compare our model solutions with observations of the central regions $\left(r<0.25 r_{\text {vir }}\right.$, where $r_{\mathrm{vir}}=c r_{s}$ is the virial radius) of eight clusters: Virgo, Abell 262, Sersic 159-03, Abell 4059, Hydra A, Abell 496, Abell 1795, and Perseus. Temperature and hydrogen-number-density $\left(n_{H}\right)$ profiles for these clusters are taken from table 5 of Kaastra et al (2004). Redshifts ( $z$ ) and angulardiameter distances $d_{\text {scdm }}$ are given in table 1 of Kaastra et al (2004). The data of Kaastra et al (2004) are obtained assuming a standard cold dark matter (SCDM) cosmology with $\Omega=1$ and $H_{0}=50 \mathrm{~km} \mathrm{~s}^{-1} \mathrm{Mpc}^{-1}$. We convert to a $\Lambda \mathrm{CDM}$ cosmology with $\Omega_{0}=0.3, \Omega_{\Lambda, 0}=0.7$, and $H_{0}=70 \mathrm{~km} \mathrm{~s}^{-1} \mathrm{Mpc}^{-1}$ by calculating the ratio of angular-diameter distance in the two cosmologies, $\zeta(z) \equiv d_{\text {scdm }} / d_{\Lambda \text { cdm }}$, for each cluster in the sample. We then multiply Kaastra et al's (2004) values for $n_{H}$ by $\sqrt{\zeta}$ (since the observed X-ray flux and angular size are fixed) and multiply linear distances by $\zeta^{-1}$. Values of $\zeta$, as well as the cooling radius, are given in table 2. [The conversion from $n_{H}$ to $n_{e}$ is given by equation (21).]

We adjust a single parameter, $r_{\text {source }}$, to fit to the observations. The optimal values for $r_{\text {source }}$ are given in table 3. The temperature and density profiles in the model solutions are plotted in figures 1 and 2. We note that the central peak in the Perseus temperature data is due to the hard power-law spectrum of the central active galaxy NGC 1275 (E. Churazov, private communication). The values of $\dot{M}$ and $L_{\mathrm{cr}}$, as well as fluid quantities at $r_{1}=0.2 \mathrm{kpc}$ and $r_{\text {outer }}$ are given in table 3 , The radial profiles of the rms turbulent velocity and cosmic-ray pressure are presented in appendix $\mathrm{A}$.

Overall, the model profiles match the observations quite well, which suggests that convection is an important process in cluster cores. The model, however, substantially underestimates the ob-

served density in Sersic 159-03 at $r \lesssim 50 \mathrm{kpc}$. This discrepancy may be explained by a recent study by Werner et al (2007). These authors found that Sersic 159-03 has the largest soft x-ray excess of all clusters observed by XMM-Newton and argued that the observed excess is best explained by 
Table 2: Cluster parameters

\begin{tabular}{lrr}
\hline \hline Cluster & $\frac{d_{\text {scdm }}}{d_{\Lambda \mathrm{cdm}}}$ & $\begin{array}{r}r_{\text {cool }} \\
(\mathrm{kpc})\end{array}$ \\
\hline \hline Virgo & & \\
Abell & 1.0 & 73 \\
Sersic 159-03 & 1.39 & 61 \\
Abell 4059 & 1.37 & 86 \\
Hydra A & 1.36 & 130 \\
Abell 496 & 1.38 & 89 \\
Abell 1795 & 1.36 & 130 \\
Perseus & 1.39 & 128 \\
\hline
\end{tabular}

The quantity $d_{\text {scdm }}$ is the angular-diameter distance to each cluster in the SCDM cosmology employed by Kaastra et al (2004) in which $\Omega=1$ and $H_{0}=50 \mathrm{~km} \mathrm{~s}^{-1} \mathrm{Mpc}^{-1} . d_{\Lambda \mathrm{cdm}}$ is angular-diameter distance to each cluster in the $\Lambda \mathrm{CDM}$ cosmology assumed in this paper, in which $\Omega=0.7, \Omega_{\Lambda}=0.3$ and $H_{0}=70 \mathrm{~km} \mathrm{~s}^{-1} \mathrm{Mpc}^{-1}$. For Virgo, $d_{\mathrm{scdm}} / d_{\Lambda \mathrm{cdm}}$ is set equal to 1.0 , since we use the same distance $(16 \mathrm{Mpc})$ to Virgo as employed by Kaastra et al (2004). The cooling radii $r_{\text {cool }}$ are taken from Kaastra et al (2004) but rescaled to $\Lambda$ CDM. Kaastra et al's values of $r_{\text {cool }}$ are the radii at which the radiative cooling time $t_{\text {cool }}$ is $15 \mathrm{Gyr}$ in SCDM. At the rescaled values of $r_{\text {cool }}, t_{\text {cool }}$ is $15 \mathrm{Gyr} \cdot\left(d_{\Lambda \mathrm{cdm}} / d_{\mathrm{scdm}}\right)^{1 / 2}$ in $\Lambda \mathrm{CDM}$ (because the cooling time scales like $n_{e}^{-1}$ and $n_{e} \propto d^{-1 / 2}$ for a fixed observed X-ray flux and angular size, where $d$ is the angular-diameter distance).

the presence of a substantial population of non-thermal electrons that is concentrated in the cluster core. When they modeled the observed emission as coming from a combination of thermal plasma and nonthermal electrons, they found that the x-ray emission between 0.3 and $10 \mathrm{keV}$ from nonthermal electrons is a substantial fraction of the emission from the thermal plasma at large radii $(\sim 35-55 \%$ at $r \simeq 375 \mathrm{kpc})$ but only a small fraction of the emission at small radii $(\sim 1-7 \%$ at $r \lesssim 50 \mathrm{kpc}$ ). Thus, the non-thermal contribution to the emission measure does not lead directly to a large change in the observationally inferred electron density at $r \lesssim 50 \mathrm{kpc}$. On the other hand, Werner et al (2007) note that if there is a non-thermal proton population with significantly more pressure than the non-thermal electrons, the total cluster mass may be significantly underestimated. Moreover, since the non-thermal pressure inferred by Werner et al (2007) peaks strongly towards the cluster's center, the actual gravitational acceleration in the central 50-100 kpc may be much larger than in an NFW profile calculated neglecting non-thermal pressure. This is an issue for all the clusters that we consider, but especially for Sersic 159-03, since its especially large soft excess indicates a large non-thermal pressure fraction. We note that although the non-thermal emission 
is less peaked than the thermal emission in the results of Werner et al (2007) (i.e., $p_{\text {non-thermal }} / n_{e}^{2}$ decreases towards the center), the non-thermal pressure is more peaked than the thermal pressure ( $p_{\text {non-thermal }} / p$ increases inwards). If we were to re-calculate our model solutions using a larger gravitational acceleration in the cluster core, the thermal plasma density would peak more sharply near the cluster center than in Figure 1. Thus, the deviation between the model and observations of Sersic 159-03 may be due to a significant underestimate of the gravitational acceleration in this cluster resulting from its unusually large non-thermal pressure.

Table 3: Physical quantities in the model solutions

\begin{tabular}{|c|c|c|c|c|c|c|c|c|c|c|}
\hline Cluster & $\begin{array}{l}r_{\text {source }} \\
(\mathrm{kpc})\end{array}$ & $\begin{array}{l}n_{\mathrm{e}}\left(r_{1}\right) \\
\left(\mathrm{cm}^{-3}\right)\end{array}$ & $\begin{array}{c}k_{\mathrm{B}} T\left(r_{1}\right) \\
(\mathrm{keV})\end{array}$ & $\frac{p_{\mathrm{cr}}\left(r_{1}\right)}{p\left(r_{1}\right)}$ & $\frac{\left|\left\langle v_{r}\left(r_{1}\right)\right\rangle\right|}{c_{s}\left(r_{1}\right)}$ & $\begin{array}{c}\dot{M}_{\text {Bondi }} \\
\left(M_{\odot} \mathrm{yr}^{-1}\right)\end{array}$ & $\begin{array}{c}L_{\mathrm{cr}} \\
(\mathrm{erg} / \mathrm{s})\end{array}$ & $\begin{array}{l}r_{\text {outer }} \\
(\mathrm{kpc})\end{array}$ & $\begin{array}{c}k_{B} T_{\text {outer }} \\
(\mathrm{keV})\end{array}$ & $\begin{array}{l}n_{\mathrm{e}, \text { outer }} \\
\left(\mathrm{cm}^{-3}\right)\end{array}$ \\
\hline Virgo & 1.70 & 0.115 & 1.27 & 0.268 & 0.00207 & 0.00203 & $5.77 \times 10^{41}$ & 49.3 & 2.50 & $2.85 \times 10^{-3}$ \\
\hline Abell 262 & 34.0 & 0.231 & 0.118 & 0.153 & 0.0388 & 0.0232 & $6.59 \times 10^{42}$ & 93.6 & 2.16 & $1.22 \times 10^{-3}$ \\
\hline Sersic $159-03$ & 46.0 & 0.397 & 0.133 & 1.24 & 0.365 & 0.399 & $1.13 \times 10^{44}$ & 165 & 2.38 & $1.35 \times 10^{-3}$ \\
\hline Abell 4059 & 24.0 & 0.168 & 0.727 & 0.156 & 0.0562 & 0.0607 & $1.72 \times 10^{43}$ & 137 & 3.89 & $1.75 \times 10^{-3}$ \\
\hline Hydra A & 35.0 & 0.742 & 0.340 & 0.740 & 0.257 & 0.839 & $2.38 \times 10^{44}$ & 160 & 3.28 & $2.05 \times 10^{-3}$ \\
\hline Abell 496 & 5.00 & 0.0437 & 0.965 & 0.701 & 0.0201 & 0.00650 & $1.85 \times 10^{42}$ & 96.2 & 3.93 & $2.53 \times 10^{-3}$ \\
\hline Abell 1795 & 40.0 & 0.671 & 0.165 & 0.700 & 0.177 & 0.363 & $1.03 \times 10^{44}$ & 184 & 5.56 & $2.19 \times 10^{-3}$ \\
\hline Perseus & 16.0 & 2.47 & 0.487 & 0.121 & 0.0264 & 0.343 & $9.75 \times 10^{43}$ & 162 & 5.27 & $2.16 \times 10^{-3}$ \\
\hline
\end{tabular}

$r_{\text {source }}$ is the size of the cosmic-ray acceleration region that leads to the best fit between the mixing-length model and the observations of Kaastra et al (2004). $n_{\mathrm{e}}\left(r_{1}\right), T\left(r_{1}\right), p\left(r_{1}\right)$, and $p_{\text {cr }}\left(r_{1}\right)$ are the electron density, temperature, thermal pressure, and cosmic-ray pressure at the inner radius $r_{1}=0.2 \mathrm{kpc} . v_{r}$ and $c_{\mathrm{s}}$ are the radial velocity and adiabatic sound speed. $\dot{M}_{\text {Bondi }}$ is the Bondi accretion rate based on the plasma density and plasma temperature at $r=r_{1}$, and $L_{\mathrm{cr}}=0.005 \dot{M}_{\mathrm{Bondi}} c^{2}$ is the cosmic-ray luminosity of the central radio source. $T_{\text {outer }}$ and $n_{\mathrm{e}, \text { outer }}$ are the observed temperature and electron density at the radius $r_{\text {outer }}$ of the outer boundary used in our shooting method.

One of the quantities that is calculated as part of our solutions is the cosmic-ray luminosity $L_{\mathrm{cr}}$ of the central AGN. To further test the plausibility of our model, we compare the theoretically predicted values of $L_{\mathrm{cr}}$ from table 3 and the observationally inferred mechanical luminosity $L_{\text {mech }}$ of the central AGN in the six clusters in our sample for which we were able to find published values. The mechanical luminosities are taken from Bîrzan et al (2004), and are calculated from observations of X-ray cavities, by assuming that an energy $p V$ (where $p$ is the surrounding pressure and $V$ is the cavity volume) per cavity is released during a time equal to the buoyancy time scale. We plot $L_{\text {mech }}$ versus $L_{\mathrm{cr}}$ in figure 3. The error bars in this figure take into account projection effects on the estimate of the cavity volume as well as uncertainties in the ages of the cavities. These two 

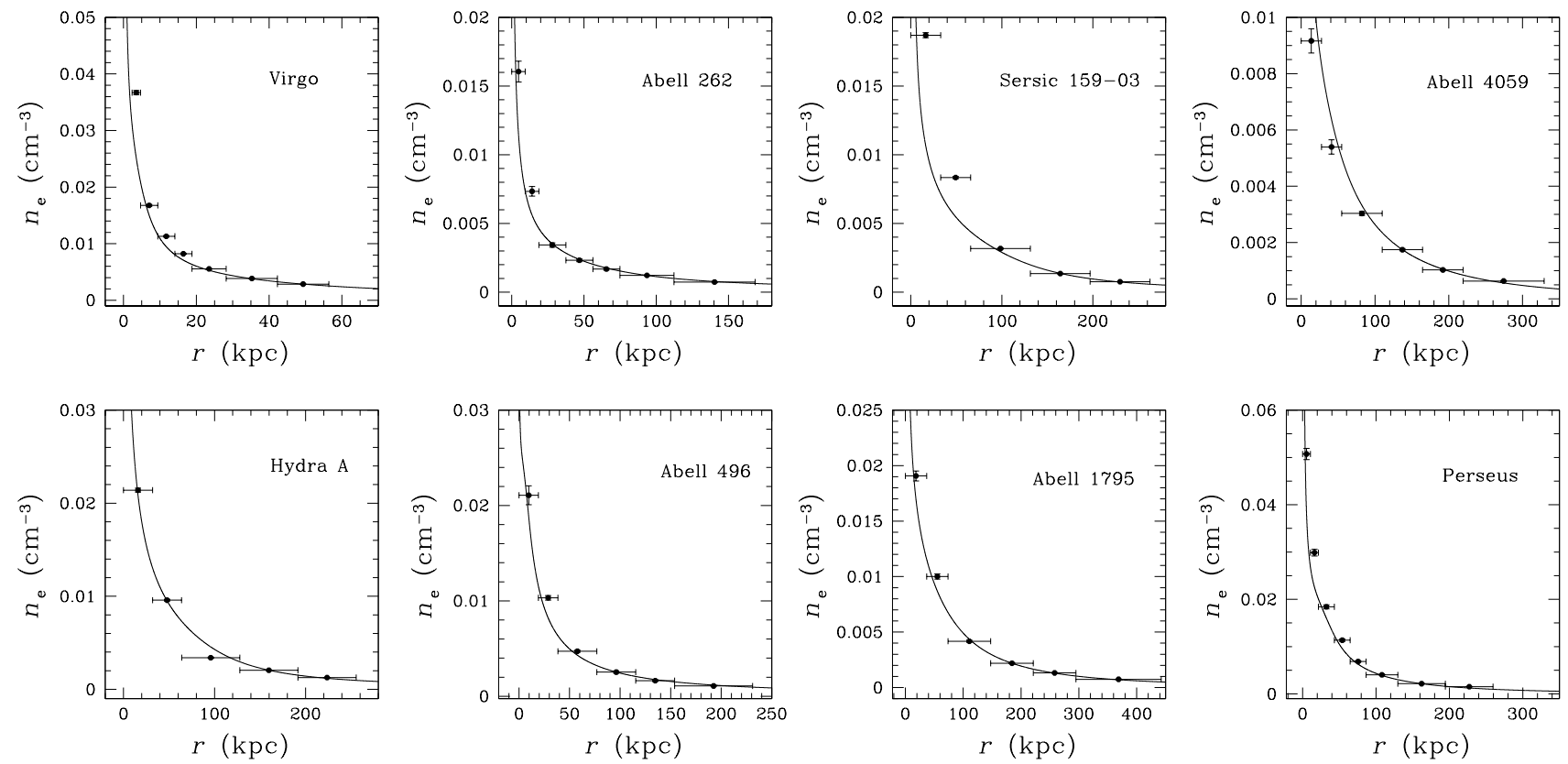

Fig. 1. - The solid lines give the electron density as a function of radius in our model solutions for the eight clusters in our sample. The data points are from the observations of Kaastra et al (2004).

luminosities are strongly correlated over a range of $\sim 100-1000$ in luminosity.

\section{The energy budget of the intracluster medium}

In our model, radiative cooling is balanced by a combination of thermal conduction, convective heating, and radial inflow due to the accretion onto the central AGN. To distinguish between these last two mechanisms, we separate the average radial velocity into two components,

$$
\left\langle v_{r}\right\rangle=v_{\text {inflow }}+v_{r \text { turb }}
$$

where

$$
v_{\text {inflow }}=-\frac{\dot{M}}{4 \pi r^{2}\langle\rho\rangle}
$$

is the inflow rate that arises in a laminar radial flow with constant mass accretion rate $\dot{M}$. The term $v_{r, \text { turb }}$ is an additional average radial velocity that is induced by the convection. [Its value is given by $-\left\langle\delta \rho \delta v_{r}\right\rangle /\langle\rho\rangle$, as in equation (B5).] With this definition in hand, we write the average of equation (4), divided by $(\gamma-1)$, as

$$
0=\left\langle H_{\text {inflow }}+H_{\text {conv }}+H_{\text {visc }}+H_{\text {tc }}-R\right\rangle .
$$



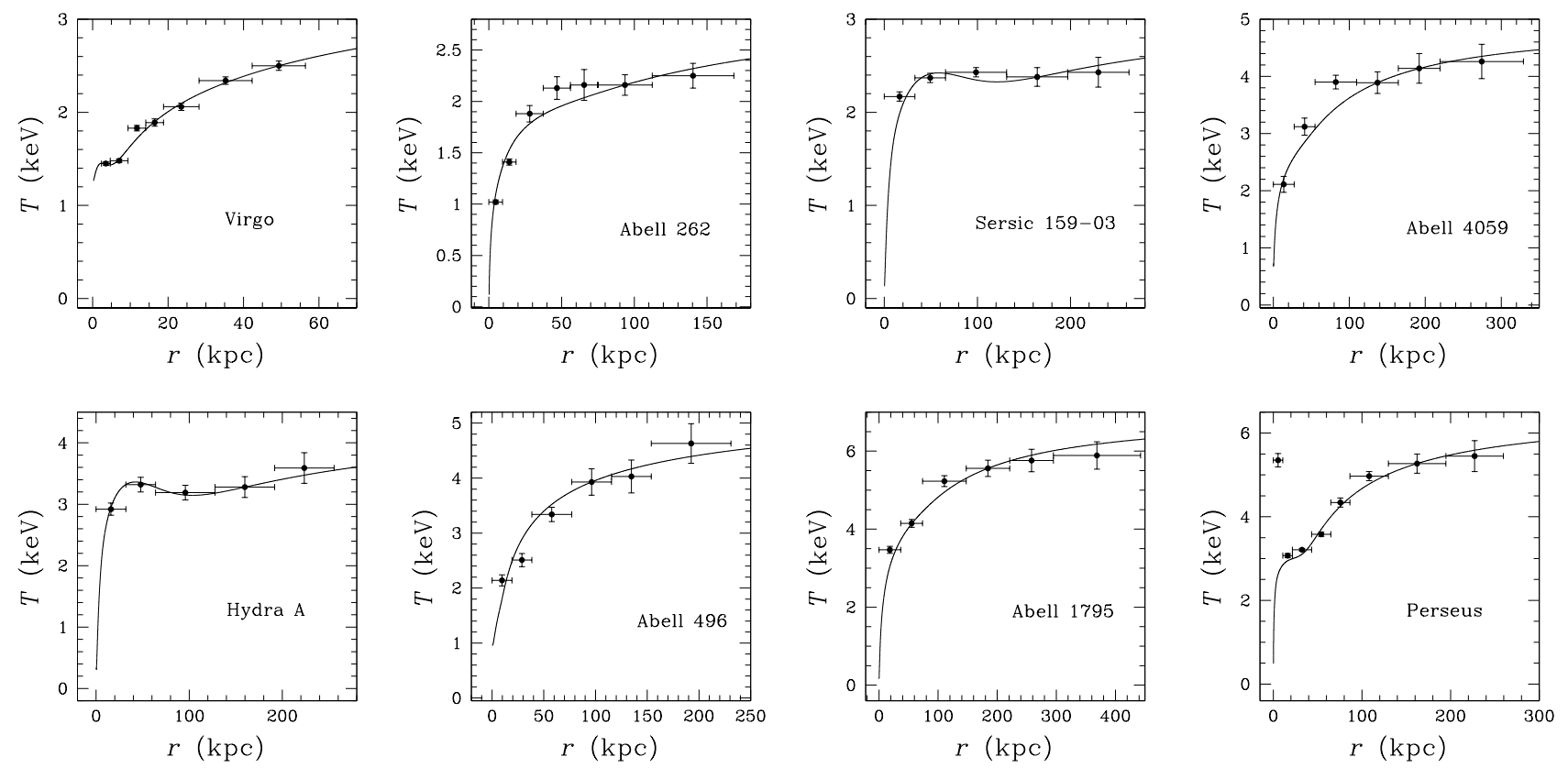

Fig. 2.- The solid lines give the temperature as a function of radius in our model solutions for the eight clusters in our sample. The data points are from the observations of Kaastra et al (2004).

Here,

$$
\left\langle H_{\text {inflow }}\right\rangle=-\frac{1}{(\gamma-1) r^{2}} \frac{d}{d r}\left(r^{2} v_{\text {inflow }}\langle p\rangle\right)-\frac{\langle p\rangle}{r^{2}} \frac{d}{d r}\left(r^{2} v_{\text {inflow }}\right)
$$

is the source term associated with $v_{\text {inflow. }}$ The term

$$
\left\langle H_{\text {conv }}\right\rangle=\left\langle-\frac{\nabla \cdot(v p)}{(\gamma-1)}-p \nabla \cdot v-H_{\text {inflow }}\right\rangle
$$

is the convective heating rate of the thermal plasma, excluding viscous dissipation. It includes the turbulent diffusion of heat as well as the turbulent $p d V$ work done on the thermal plasma by cosmic rays. The average of the viscous dissipation term is set equal to

$$
\left\langle H_{\mathrm{visc}}\right\rangle=\frac{0.42 \rho u_{\mathrm{rms}}^{3}}{l},
$$

where $l=0.4 r$ is the mixing length, $u_{\mathrm{rms}}$ is the rms turbulent velocity defined in equation (B71), and the constant 0.42 is taken from direct numerical simulations of compressible magnetohydrodynamic turbulence (Haugen, Brandenburg, \& Dobler 2004) 1 The average of $H_{\mathrm{tc}}$ is given by equation (26), and the average of $R$ is given by equation (17).

\footnotetext{
${ }^{1}$ The constant 0.42 is obtained by taking the mixing length $l$ to correspond to $\pi / k_{\mathrm{p}}$ in the simulations of Haugen et al (2004), where $k_{\mathrm{p}}$ is the wave number at which $k E(k)$ peaks, and $E(k)$ is the power spectrum of the turbulent velocity.
} 


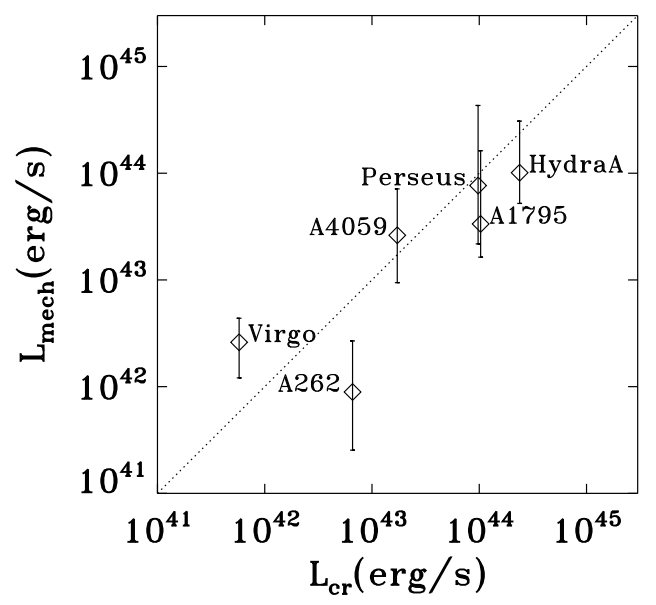

Fig. 3.- Comparison between the cosmic-ray luminosities in our model calculations $L_{\mathrm{cr}}$ against observationally inferred values of the mechanical luminosities of the central AGN in six of the clusters in our sample (Bîrzan et al 2004). The dotted line represents equality between these two quantities.

In figure 4 , we plot the averages of $H_{\text {inflow }}$ (dotted line), $H_{\text {conv }}$ (long-dashed line), $H_{\text {tc }}$ (shortdashed line), and $R$ (solid line), integrated over volume from the inner radius of our model $\left(r_{1}=\right.$ $0.2 \mathrm{kpc}$ ) out to radius $r$. We find that $\left\langle H_{\mathrm{visc}}\right\rangle \ll\left\langle H_{\text {conv }}\right\rangle$ everywhere in each cluster, and so we omit $H_{\text {visc }}$ from the figures to keep the plots easier to read 2

We can divide the clusters into two groups, those with AGN-dominated heating and those with conduction-dominated heating. In Hydra A and Sersic 159-03, the heating within the central $100 \mathrm{kpc}$ is dominated by convection driven by the central AGN. In all of the other clusters, the heating within the central $100 \mathrm{kpc}$ is dominated by thermal conduction. The inability of conduction to balance cooling in Hydra A and Sersic 159-03 was previously noted by Zakamska \& Narayan (2003). These authors constructed density and temperature profiles for clusters assuming that radiative cooling is balanced by conductive heating, setting the thermal conductivity equal to a constant $f_{c}$ times the Spitzer thermal conductivity. For Hydra A and Sersic 159-03, they found that the values of $f_{c}$ that best fit the observations were 1.5 and 5.6, respectively, much larger than the theoretically expected value of $f_{c} \simeq 0.1-0.2$. In contrast, for Abell 1795 the best-fit value of $f_{c}$ was 0.2 .

\footnotetext{
${ }^{2}$ We note that equation (38) is not exactly satisfied by our model solutions. In our model, we use the total-energy equation [equation (B3)] instead of the plasma energy [equation (4)]. Although equation (4) is exactly satisfied when equations (B3), (3), and (5) are satisfied, our mixing-length approximation of the average of equation (4) is not exactly satisfied when our mixing-length approximations to the averages of equations (B3), (3), and (5) are satisfied. This discrepancy is noticeable at the largest radii in Hydra A and Sersic 159-03.
} 

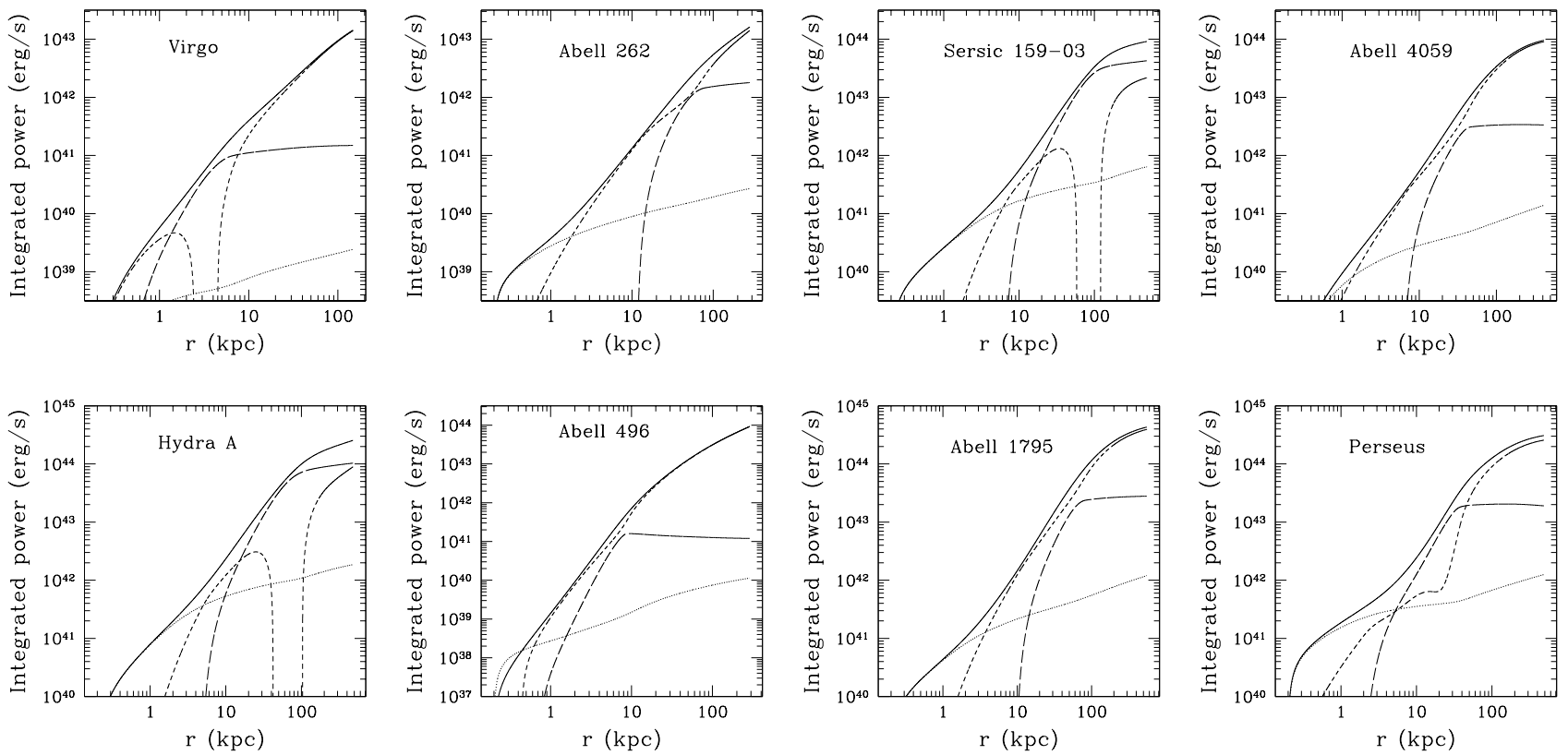

Fig. 4. - The energy sources and sinks in the thermal plasma, integrated over volume from the inner radius $r_{1}=$ $0.2 \mathrm{kpc}$ out to radius $r$. The solid line is the radiative losses (X-ray luminosity), the short dashed line is the heating from thermal conduction, the dotted line is power contributed by the inflow associated with the mass accretion rate, and the long-dashed line is the heating power due to the convective turbulence, which includes both the turbulent diffusion of heat and $p d V$ work by cosmic-rays.

What are the characteristics of a cluster that determine whether AGN feedback or thermal conduction is the dominant heat source within the central $100 \mathrm{kpc}$ ? Given that $\kappa_{S} \propto T^{5 / 2}$, it seems clear that a lower average temperature $T_{\text {avg }}$ makes thermal conduction less able to balance radiative cooling, leading in turn to a relatively greater role for AGN feedback. However, although $T_{\text {avg }}$ is important for determining the relative strength of conduction and AGN feedback, on its own the value of $T_{\text {avg }}$ does not explain our results, since Virgo and Abell 262 have temperatures comparable to that of Sersic 159-03 and lower than that of Hydra A. An equally important factor appears to be the baryon density in the core. In particular, the clusters with AGN-dominated heating are significantly denser at a given radius than clusters with conduction-dominated heating that have similar average temperatures. For example, for radii between 20 and $50 \mathrm{kpc}$, the electron density in Sersic 159-03 is 2-3 times greater than in Virgo. Similarly, at $r=100 \mathrm{kpc}$ the electron density in Hydra $\mathrm{A}$ is $~ 60 \%$ larger than in Abell 4059.3 even though Abell 4059 has a higher average temperature and larger virial mass. (Typically, at a fixed radius the density is larger in hotter,

\footnotetext{
${ }^{3}$ This ratio is based on the model density profile due to the offset in the radius of the observed densities, but a similar conclusion is reached by interpolating between data points.
} 
more massive clusters.) It thus appears that the clusters in which AGN feedback dominates most strongly over conduction are those in which the clusters' ongoing formation channels unusually large quantities of baryons towards the clusters' cores.

We conclude this section with a few additional comments relating to figures 3 and 4 Bîrzan et al (2004) found that their observationally inferred values of $L_{\text {mech }}$ for 16 clusters were correlated with the X-ray luminosity inside the cooling radius $r_{\text {cool }}$, denoted $L_{X}$, supporting the idea that AGN feedback is at least part of the solution to the cooling-flow problem. However, the level of the mechanical luminosity in their study turns out to be a factor of 1 to 20 lower than the X-ray luminosity, which raises the question of whether the mechanical luminosity is sufficient to offset cooling in these clusters. Our model solutions and figure 3 show that the mechanical luminosity is indeed sufficient when thermal conduction is also accounted for, and our discussion above regarding AGN-dominated heating versus conduction-dominated heating offers an explanation for the large variations in the ratio $L_{X} / L_{\text {mech }}$. When heating is dominated by conduction, AGN feedback heating is only a small fraction of the power radiated from within the cooling radius, and $L_{X} / L_{\text {mech }}$ is large. On the other hand, when AGN-driven convection dominates the heating, the AGN heating power is similar to the total power radiated from within $r_{\text {cool }}$, and $L_{X} \sim L_{\text {mech }}$.

We note that figure 4 shows that in Hydra A, Sersic 159-03, and Virgo, conduction actually acts to cool the plasma over a limited range of $r$ due to the local maximum in the temperature profile. Also, in none of the clusters is the total convective heating rate equal to $L_{\mathrm{cr}}$. This is because the cosmic-ray luminosity is the power deposited into the cosmic-ray fluid, and only part of this is transferred to the thermal plasma through $p d V$ work. Additional plasma heating arises from the redistribution (turbulent diffusion) of plasma thermal energy resulting from the convective motions. As can be seen from figure 4 and table 3, the total convective heating of the thermal plasma is typically on the order of one-third of $L_{\mathrm{cr}}$.

\section{Clusters with central cooling flows}

An important point to emerge from figure 4 is the appearance of central cooling flows in several clusters - Abell 262, Sersic 159-03, Hydra A, Abell 1795, and Perseus - with radii $r_{\mathrm{cf}}$ that are typically a few kpc. Heating is unable to balance cooling at $r<r_{\mathrm{cf}}$ in these clusters for several reasons: the AGN feedback heating is distributed over a large volume, thermal conduction becomes less efficient at small $r$ due to the lower temperatures and the fact that $\kappa_{T} \propto T^{5 / 2}$, and the radiative losses per unit volume peak sharply at small $r$ due to the large plasma densities. As a result, a cooling flow develops in which the energy lost to cooling is replenished by the inflow. Within this central region, we have the approximate relation $t_{\text {cool }} \sim t_{\text {inflow }}$, where $t_{\text {inflow }}=r /\left|v_{\text {inflow }}\right|$ is the inflow time and $t_{\text {cool }}=1.5 n k_{B} T / R$ is the local cooling time. Because $t_{\text {inflow }} \sim t_{\text {cool }}$, the mass 
accretion rate at $r=r_{\mathrm{cf}}$ is approximately given by

$$
\dot{M} \sim 4 \pi r_{\mathrm{cf}}^{2} \rho\left(r_{\mathrm{cf}}\right)\left[\frac{r_{\mathrm{cf}}}{t_{\mathrm{cool}}\left(r_{\mathrm{cf}}\right)}\right] \sim \frac{M_{\mathrm{cf}}}{t_{\mathrm{cool}}\left(r_{\mathrm{cf}}\right)},
$$

where $r_{\mathrm{cf}}$ is the radius of the central cooling flow region, and $M_{\mathrm{cf}}$ is the mass of plasma contained within the cooling flow region. Because we have no sources or sinks of plasma, $\dot{M}$ is independent of $r$ in our model.

How do these central cooling flows match onto adiabatic Bondi flow at smaller radii? In our model, as $r$ decreases from $r_{\text {cf }}$ towards zero, $\rho$ rises and $T$ decreases until the Bondi accretion rate at the fixed radius $r_{1}=0.2 \mathrm{kpc}$ matches the cooling-flow mass accretion rate given by equation (42). However, our forcing the flow to become adiabatic at $r_{1}$ is artificial, and leads to an unrealistic plasma profile near $r_{1}$ with an abrupt transition in the flow at $r_{1}$. A better approach was adopted by Quataert \& Narayan (2000). These authors investigated radial inflow with cooling in the absence of thermal conduction and cosmic rays using a numerical shooting method and solved all the way in to the sonic point, $r=r_{\text {sonic }}$, at which $v_{r}=-c_{s}$. They found a smooth transition from an outer cooling flow with $t_{\text {inflow }} \simeq t_{\text {cool }}$ to an inner adiabatic Bondi flow with $t_{\text {inflow }} \ll t_{\text {cool }}$, provided that $r_{\text {sonic }} \lesssim r_{\text {tr }}$, where

$$
r_{\mathrm{tr}} \equiv \frac{G M_{\mathrm{bh}}}{\sigma^{2}}=0.05 \mathrm{kpc}\left(\frac{M_{\mathrm{bh}}}{10^{9} M_{\odot}}\right)\left(\frac{\sigma}{300 \mathrm{~km} / \mathrm{s}}\right)^{-2}
$$

is the radius within which gravity is dominated by the black hole and $\sigma$ is the circular velocity of the BCG, which was taken to be independent of $r$. They also found that equation (42) was an accurate estimate of the numerically calculated mass accretion rate in the absence of mass dropout. For $r_{\text {sonic }}<r_{\text {tr }}$, Quataert \& Narayan's solution satisfies $c_{\mathrm{s}} \sim \sigma=$ constant at $r>r_{\text {tr }}$. In the absence

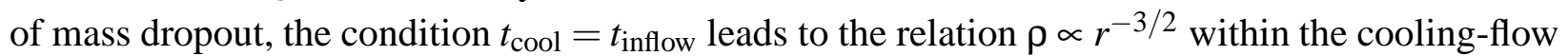
part of their solution. The Bondi accretion rate $\dot{M}_{\text {Bondi }}$ [given by equation (30)] evaluated at a radius $r$ within the cooling-flow part of their solution thus increases towards smaller $r$ like $r^{-3 / 2}$. At a sufficiently small value of $r$, which we call $r_{\text {ad }}$, the Bondi accretion rate equals the rate $\dot{M}_{\text {cf }}$ at which mass flows in through the cooling flow, and the flow makes a transition to an adiabatic Bondi flow. At $r<r_{\text {ad }}$, the ratio $t_{\text {cool }} / t_{\text {inflow }}$ increases towards smaller $r$, and so the neglect of cooling at $r<r_{\text {ad }}$ is self-consistent. We note that the Bondi accretion formula can be applied in the model of Quataert \& Narayan (2000) at the outer boundary of the adiabatic flow region, $r_{\mathrm{ad}}$, even if $r_{\text {ad }}$ lies outside the region in which the black hole dominates the gravitational acceleration. This is because the Bondi accretion rate depends only on the specific entropy $s$ of the plasma and $M_{\mathrm{bh}}$, and $s$ is constant for $r<r_{\text {ad }}$.

It would be valuable to incorporate into our model an approach similar to that of Quataert \& Narayan (2000), including cosmic rays, thermal conduction, and the possibility of convection. Although such a calculation is beyond the scope of this paper, we expect that in such an analysis 
the mass accretion rate of the central accretion flow and the plasma parameters at $r_{\text {Bondi }}$ are still controlled by $\dot{M}_{\text {cf }}$, as in Quataert \& Narayan's (2000) work. Because the central accretion flow is in some sense slaved to the surrounding cooling flow, the AGN regulates the mass accretion rate primarily by controlling the properties of the central cooling flow, and in particular by controlling $r_{\mathrm{cf}}$. For example, if $\dot{M}$ rises above the equilibrium value, the AGN-feedback heating rises. This then reduces $r_{\mathrm{cf}}$, because one has to go to smaller $r$ in order for $\rho$ to rise enough that cooling exceeds the convective heating rate. The reduction in $r_{\mathrm{cf}}$ reduces $\dot{M}$, as can be seen from equation (42), which then causes $\dot{M}$ to drop back down to its equilibrium level.

As mentioned above, the existence of a smooth transition from a cooling flow to an inner adiabatic Bondi flow requires that $r_{\text {sonic }}<r_{\text {tr }}$. Otherwise, as described by Quataert \& Narayan (2000), the ratio $t_{\text {cool }} / t_{\text {inflow }}$ decreases inwards in the supersonic region at $r_{\mathrm{tr}}<r<r_{\text {sonic }}$, and the plasma cools rapidly to very low temperature. In this case, the cooling plasma could still end up fueling the central black hole, but it would do so through some process other than the one we have assumed in our model, e.g., by forming stars whose winds then feed the black hole or through infalling cold gas [see, e.g., Pizzolato \& Soker (2005) and Soker (2006)].

Under what conditions is $r_{\text {sonic }}>r_{\text {tr }}$ ? One factor that can cause $r_{\text {sonic }}$ to exceed $r_{\text {tr }}$ is a small central black hole mass, since a smaller $M_{\text {bh }}$ reduces $r_{\text {tr }}$. In addition, as illustrated in Quataert $\&$ Narayan's approximate analytic results, if the black hole's contribution to gravity were hypothetically ignored, $r_{\text {sonic }}$ would increase with increasing $\dot{M}$. Thus, a sufficiently large $\dot{M}$ can also cause $r_{\text {sonic }}$ to exceed $r_{\text {tr }}$. A large $\dot{M}$ results from either a large $L_{\text {cr }}$ or a small accretion efficiency $\eta$. As discussed in the previous section, $L_{\mathrm{cr}}$ is approximately determined by the baryon density and temperature at the cooling radius - a higher density and/or lower temperature at $r_{\text {cool }}$ means that thermal conduction can offset less of the radiative cooling within the cooling radius, which in turn leads to a larger $L_{\mathrm{cr}}$. Thus, to summarize, smaller values of $M_{\mathrm{bh}}, \eta$, or $T\left(r_{\mathrm{cool}}\right)$ and/or larger values of $\rho\left(r_{\text {cool }}\right)$ can cause $r_{\text {sonic }}$ to exceed $r_{\text {tr }}$, preventing a smooth transition from a cooling flow to an inner Bondi flow, and causing the cooling of intracluster plasma to low temperatures at $r>r_{\text {tr }}$.

We note that if the accretion efficiency $\eta_{\text {cool }}$ that arises when plasma cools to low temperature outside $r_{\text {tr }}$ is much smaller than the accretion efficiency $\eta$ associated with Bondi accretion, then a flow that cools rapidly outside $r_{\text {tr }}$ will need a much higher $\dot{M}$ (and larger $r_{\mathrm{cf}}$ ) in order for AGN feedback to provide the heating needed to offset cooling within the cooling radius. A much larger $\dot{M}$, in conjunction with plasma cooling to low temperatures outside $r_{\text {tr }}$, would imply a much larger star formation rate within the BCG. Thus, a small $M_{\mathrm{bh}}$ or large $L_{\mathrm{cr}}$ could lead to the condition $r_{\text {sonic }}>r_{\text {tr }}$ and cause star formation at rates that significantly exceed the Bondi accretion rates listed in table 3 .

Returning to our model calculations, we list in table 3 the values of $\left|\left\langle v_{r}\right\rangle\right| / c_{s}$ at $r=r_{1}$ for the clusters in our sample. The value of $\left|\left\langle v_{r}\right\rangle\right| / c_{s}$ reaches its maximum at $r=r_{1}$ in our solutions, and 
thus our solutions satisfy $\left|\left\langle v_{r}\right\rangle\right| / c_{s}<1$ at all radii. Our calculations are thus at least marginally consistent with our assumptions of hydrostatic equilibrium and Bondi accretion. We also note that the the sound crossing time $t_{s}$ is shorter than cooling time $t_{\text {cool }}$ at all radii in our model solutions. However, our model imposes an abrupt and artificial transition in the flow at $r=0.2 \mathrm{kpc}$, which causes our solution near $r=0.2 \mathrm{kpc}$ to be inaccurate. In addition, there is significant uncertainty in the values of $M_{\mathrm{bh}}$ and $\eta$. It is thus possible that some of the clusters reach a sonic transition outside $r_{\text {tr }}$. Further investigation of this issue is needed.

\section{Summary}

There is a growing consensus that AGN feedback holds the key to solving the cooling-flow and overcooling problems for clusters of galaxies. However, the way in which an AGN's power is delivered to the diffuse intracluster plasma is still not well understood. In this paper, we suggest that an AGN's mechanical luminosity heats the intracluster plasma by accelerating cosmic rays that cause the intracluster medium to become convectively unstable. We explore this idea by developing a steady-state, mixing-length-theory model. By adjusting a single parameter in the model (the size of the cosmic-ray acceleration region, $r_{\text {source}}$ ), we obtain a good match to the observed density and temperature profiles in seven out of the eight clusters in our sample. Our model underestimates the density in the eighth cluster, Sersic 159-03, within the central $\sim 50 \mathrm{kpc}$. We suggest that this discrepancy may result from the fact that the parameters in our NFW mass model are determined neglecting the cosmic-ray pressure. At the same time, Sersic 159-03 has the largest soft x-ray excess of any cluster observed by XMM, and likely contains a large population of non-thermal particles concentrated in the cluster core. (Werner 2007) If the mass model were recalculated taking the non-thermal pressure into account, the gravitational acceleration would be larger, especially in the cluster core, which would increase the plasma density at $r \lesssim 50 \mathrm{kpc}$ in our model calculations and possibly bring the model into agreement with the observations. We also find that the cosmic-ray luminosities of the AGN in our sample are strongly correlated with the observationally inferred mechanical luminosities of these AGN. Our results suggest that AGNdriven convection is an important process in cluster cores.

In our model solutions, the radiative cooling rate is much more peaked about $r=0$ than is the rate of convective heating. As a result, a compact central cooling flow arises in our model calculations for several of the clusters in our sample. The radii, $r_{\mathrm{cf}}$, of the cooling flows are typically a few kpc. The mass accretion rate onto the central AGN in these clusters is roughly the plasma mass at $r<r_{\mathrm{cf}}$ divided by the cooling time at $r_{\mathrm{cf}}$. We suggest that the AGN regulates the mass accretion rate in these clusters by controlling $r_{\mathrm{cf}}$ : if the AGN power rises above the equilibrium level, the size of the central cooling flow decreases, the mass accretion rate drops, and 

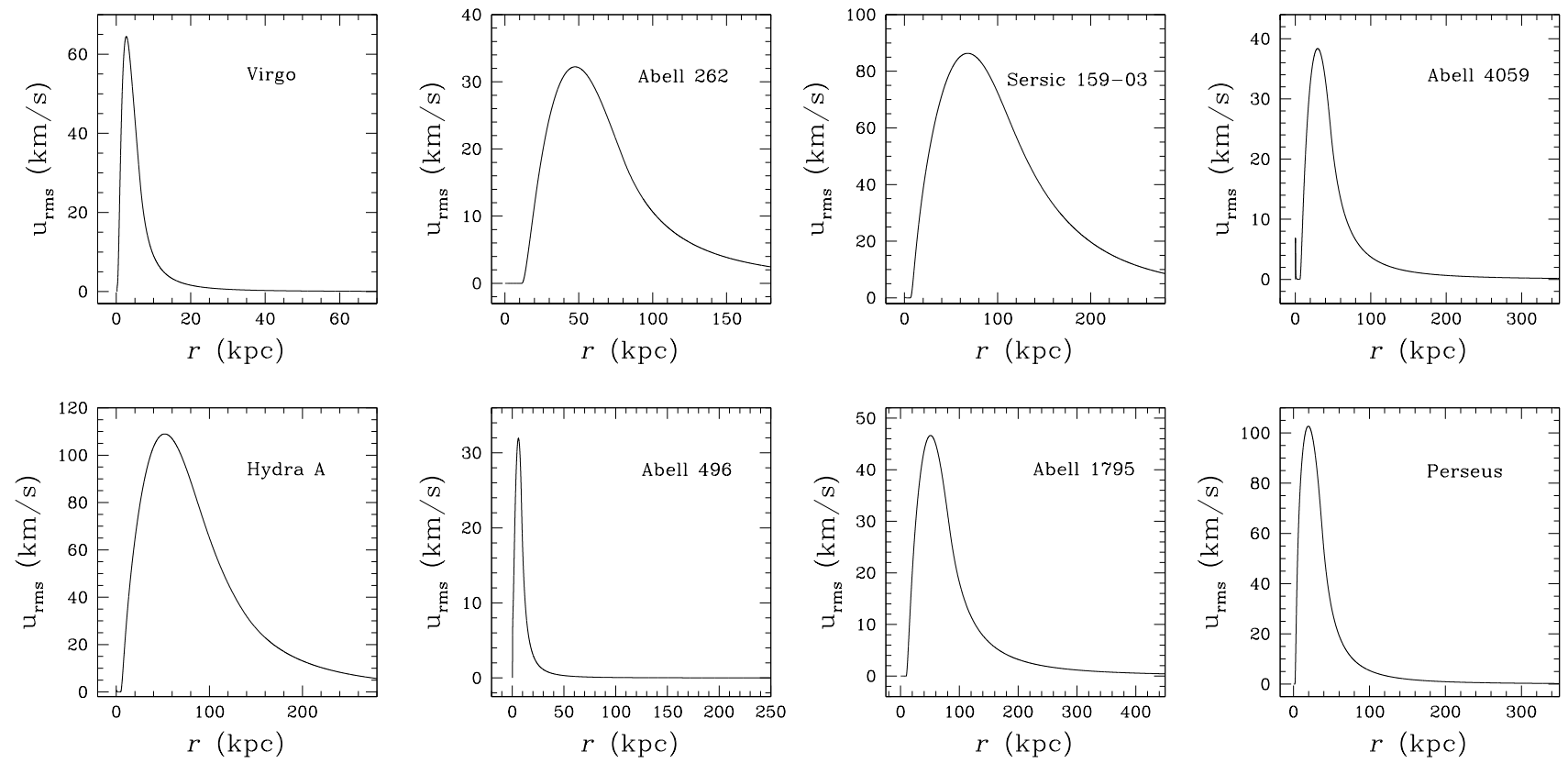

Fig. 5. - The rms turbulent velocity as a function of radius in the model solutions.

the AGN power then drops back down to the equilibrium level.

We thank Eric Blackman, Nadia Zakamska, Jelle Kaastra, and Eliot Quataert for helpful discussions. We acknowledge the usage of the HyperLeda database (http://leda.univ-lyon1.fr). This work was partially supported by NASA's Astrophysical Theory Program under grant NNG 05GH39G and by NSF under grant AST 05-49577.

\section{A. The profiles of the cosmic-ray pressure and turbulent velocity}

The profiles of the rms turbulent velocity $u_{\text {rms }}$ [defined in equation ( $(\overline{B 71})$ ] and the cosmicray pressure (as a fraction of the thermal pressure) are plotted in figures 5 and 6 . Although it is difficult to see in the cases of Sersic 159-03, Hydra A, Abell 496, and Abell 1795, figure 6 shows that $(d / d r)\left(p_{\mathrm{cr}} / p\right)>0$ at small $r$. [This can also be seen by comparing the figures with the values of $p_{\mathrm{cr}}\left(r_{1}\right) / p\left(r_{1}\right)$ listed in table 3.] This is not because $d p_{\mathrm{cr}} / d r>0$ (in fact, $d p_{\mathrm{cr}} / d r<0$ at all $r$ for each cluster), but instead because the thermal pressure decreases with radius more rapidly than the cosmic-ray pressure. We note that there was an error in one of the plotting subroutines used for paper II, which resulted in the velocities plotted in figure 4 of paper II being too large by a factor of 3. 

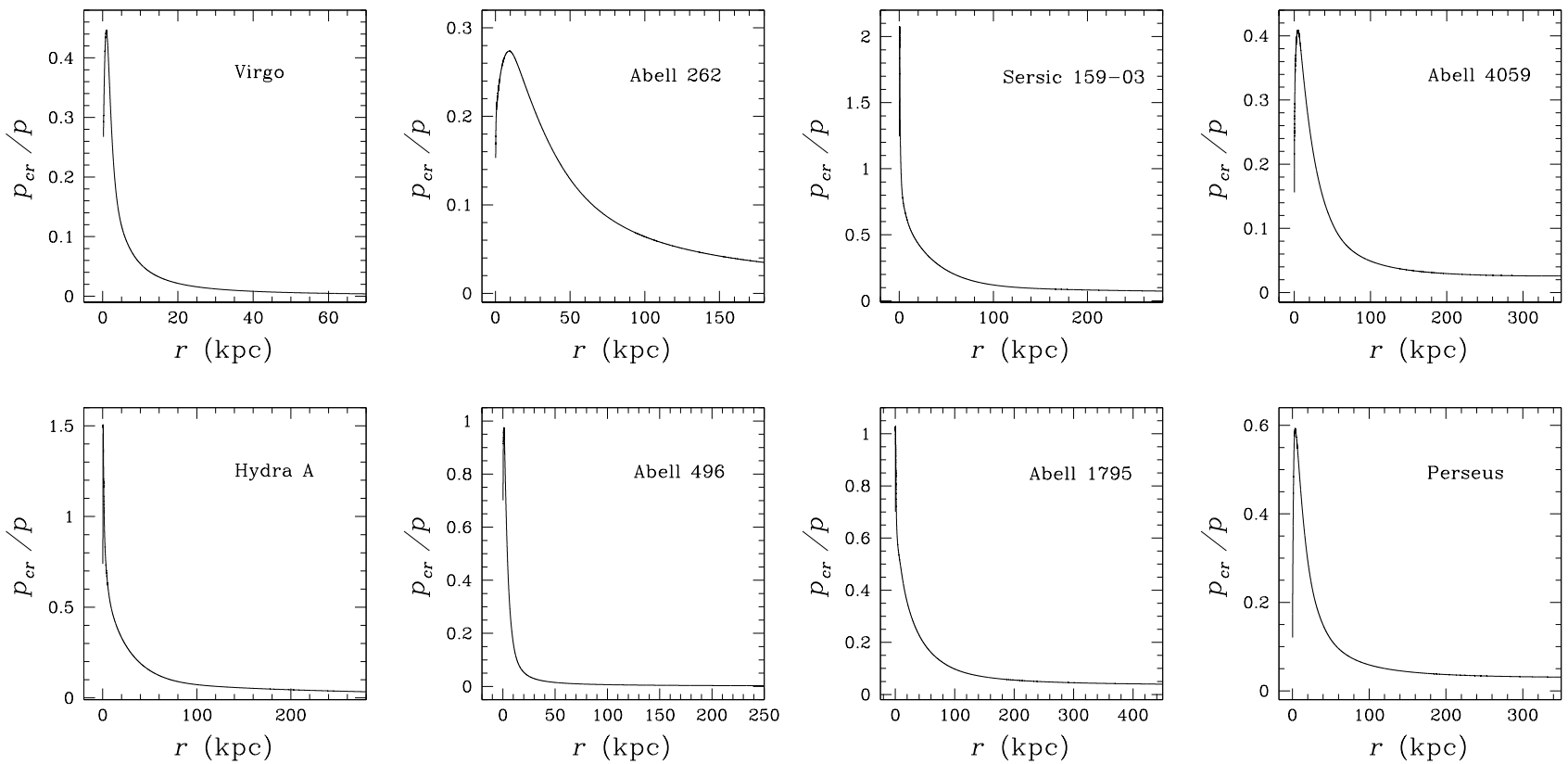

Fig. 6. - The ratio of cosmic-ray pressure to thermal pressure in the model solutions.

\section{B. The two-fluid mixing-length theory and numerical method}

In this appendix we describe the two-fluid mixing length theory that we use to model the convective intracluster medium. We take all fluid quantities to be the sum of an average value and a turbulent fluctuation, so that

$$
\begin{gathered}
\rho=\langle\rho\rangle+\delta \rho, \\
v=\langle v\rangle+\delta v,
\end{gathered}
$$

etc. We take all average quantities to depend only on the radial coordinate $r$, and we set $\langle v\rangle=\left\langle v_{r}\right\rangle \hat{r}$. We wish to solve for four quantities: $\langle\rho\rangle,\left\langle v_{r}\right\rangle,\langle T\rangle$, and $\left\langle p_{\mathrm{cr}}\right\rangle$. To do so we take the averages of four equations: equations (2), (3), and (5), as well as the total-energy equation. The latter is obtained by taking the dot product of equation (3) with $v$ and adding the resulting equation to the sum of equations (4) and (5), which yields:

$$
\begin{gathered}
\frac{\partial}{\partial t}\left(\frac{\rho v^{2}}{2}+\rho \Phi+\frac{p}{\gamma-1}+\frac{p_{\mathrm{cr}}}{\gamma_{\mathrm{cr}}-1}\right) \\
+\nabla \cdot\left(\frac{\rho v v^{2}}{2}+\rho v \Phi+\frac{\gamma v p}{\gamma-1}+\frac{\gamma_{\mathrm{cr}} v p_{\mathrm{cr}}}{\gamma_{\mathrm{cr}}-1}+\Gamma_{\mathrm{visc}}-\kappa \cdot \nabla T-\frac{\mathrm{D} \cdot \nabla p_{\mathrm{cr}}}{\gamma_{\mathrm{cr}}-1}\right) \\
=\rho \frac{\partial \Phi}{\partial t}-R+\dot{E}_{\text {source }}
\end{gathered}
$$


where $\Gamma_{\text {visc }}$ is the viscous energy flux, and where we have made use of the relation $H_{\text {visc }}-(\nabla$. $\left.\Pi_{\mathrm{visc}}\right) \cdot v=-\nabla \cdot \Gamma_{\mathrm{visc}} \cdot 4$ We assume that the viscous energy flux is much less than the advective energy flux and thus drop $\Gamma_{\text {visc. }}$. We also set $\partial \Phi / \partial t=0$.

The average of equation (2) can be written

$$
\left\langle v_{r}\right\rangle=-\frac{1}{\langle\rho\rangle}\left(Q+\frac{\dot{M}}{4 \pi r^{2}}\right)
$$

where

$$
Q \equiv\left\langle\delta \rho \delta v_{r}\right\rangle,
$$

and the mass accretion rate $\dot{M}=-4 \pi r^{2}\left\langle\rho v_{r}\right\rangle$ is a constant. The average of equation (3) yields

$$
\frac{d}{d r}\left\langle p_{\text {tot }}\right\rangle=-\langle\rho\rangle \frac{d \Phi}{d r}
$$

where

$$
p_{\text {tot }}=p+p_{\text {cr }}
$$

is the total pressure. In writing equation $(\mathrm{B} 6)$, we have taken the convection to be subsonic, so that the Reynolds stress can be neglected. We have also dropped the viscous stress, which is unimportant in the averaged equation. The average of equation (5) can be written

$$
\begin{gathered}
D_{\mathrm{cr}} \frac{d^{2}\left\langle p_{\mathrm{cr}}\right\rangle}{d r^{2}}=\frac{(1-\gamma)}{r^{2}} \frac{d}{d r}\left(r^{2} F\right)+\left(1-\gamma_{\mathrm{cr}}\right)\left(W+\left\langle\dot{E}_{\text {source }}\right\rangle\right)-\frac{d\left\langle p_{\mathrm{cr}}\right\rangle}{d r}\left[\frac{1}{r^{2}} \frac{d}{d r}\left(r^{2} D_{\mathrm{cr}}\right)\right] \\
+\frac{\gamma_{\mathrm{cr}}\left\langle p_{\mathrm{cr}}\right\rangle}{r^{2}} \frac{d}{d r}\left(r^{2}\left\langle v_{r}\right\rangle\right)+\left\langle v_{r}\right\rangle \frac{d\left\langle p_{\mathrm{cr}}\right\rangle}{d r},
\end{gathered}
$$

where

$$
F \equiv \frac{\left\langle\delta v_{r} \delta p\right\rangle}{\gamma-1}
$$

and

$$
W \equiv\langle\delta p \nabla \cdot \delta v\rangle
$$

In writing equation $(\underline{\mathrm{B} 8})$, we have again made use of the fact that the convection is subsonic, which implies that the total-pressure fluctuation is very small. As a result, we can set $\delta p \simeq-\delta p_{\mathrm{cr}}$, $\left\langle\delta v_{r} \delta p_{\mathrm{cr}}\right\rangle=-\left\langle\delta v_{r} \delta p\right\rangle$, and $\left\langle\delta p_{\mathrm{cr}} \nabla \cdot \delta v\right\rangle=-\langle\delta p \nabla \cdot \delta v\rangle$. The average of equation (B3) yields

$$
\kappa_{T} \frac{d^{2}\langle T\rangle}{d r^{2}}=Q \frac{d \Phi}{d r}+\frac{\gamma p}{(\gamma-1) r^{2}} \frac{d}{d r}\left(r^{2}\left\langle v_{r}\right\rangle\right)-\frac{\left\langle v_{r}\right\rangle}{\gamma-1}\left(\rho \frac{d \Phi}{d r}+\frac{d p_{\mathrm{cr}}}{d r}\right)+\frac{1}{r^{2}} \frac{d}{d r}\left(r^{2} F\right)
$$

\footnotetext{
${ }^{4}$ The fact that the viscous terms can be written as a total divergence reflects the fact that viscosity is neither a source of energy nor a sink of energy, but instead merely converts bulk-flow energy into thermal energy. Thus, when equation (B3) is integrated over volume, Gauss's law can be used to express the viscous terms as a surface integral, which vanishes if the boundary of the integration lies outside the plasma.
} 


$$
+W+R-\frac{d\langle T\rangle}{d r}\left[\frac{1}{r^{2}} \frac{d}{d r}\left(r^{2} \kappa_{T}\right)\right],
$$

where we have dropped the $\left\langle\rho v v^{2} / 2\right\rangle$ term since it is much smaller than the other terms for subsonic convection. In equation (B11),$\kappa_{T}$ is the average (isotropic) thermal conductivity given by equations (24) and (25), where $T$ is set equal to $\langle T\rangle$ in equation (24).

To solve equations ( $(\overline{\mathrm{B}} 4),(\mathrm{B} 6),\left([\mathrm{B} 8)\right.$, and $(\underline{\mathrm{B} 11})$ for $\langle\rho\rangle,\left\langle v_{r}\right\rangle,\langle T\rangle$, and $\left\langle p_{\mathrm{cr}}\right\rangle$, we need to express the quantities $Q, F$, and $W$ in terms of these average fluid quantities, thereby closing the equations. We accomplish this by using a two-fluid mixing length theory. Our approach is to first estimate $Q, F$, and $W$ using a local mixing length theory. In the local theory, the properties of the turbulence at some radius are determined only by the average fluid properties and gradients at that radius. We then use this local theory as the basis for a non-local mixing length theory, as in paper II. In the nonlocal theory, the properties of the turbulence at some radius $r$ are determined by a weighted average of the turbulence properties in the local mixing length theory over a range of radii. Our mixing-length theory differs from stellar mixing-length theory (Cox \& Giuli 1968) in two important ways: we include a cosmic-ray fluid, and we take into account the fact that the diffusion of heat and cosmic rays occurs almost entirely along magnetic field lines.

We derive quantities in the local mixing length theory as follows. We take the convective turbulence to have a correlation length $l$, also called the mixing length, where

$$
l=\alpha r
$$

$\alpha$ is a constant, and $r$ is the distance from the center of the cluster. Fluid parcels in convective regions are taken to rise or sink a distance $l$ before breaking up and mixing into the surrounding plasma. We take $l$ to be much smaller than the pressure scale height, so that $\alpha$ is treated as $\ll 1$. (However, as in standard mixing-length theory, after the mixing-length-theory equations are derived, we relax the requirement that $\alpha \ll 1$, and set $\alpha=0.4$ when applying the model to clusters in section 3.) We treat the fluctuations as small quantities, and take $\left\langle\sqrt{(\delta \rho)^{2}}\right\rangle /\langle\rho\rangle,\left\langle\sqrt{(\delta T)^{2}}\right\rangle /\langle T\rangle$, $\left\langle\sqrt{\left(\delta p_{\text {cr }}\right)^{2}}\right\rangle /\left\langle p_{\text {cr }}\right\rangle$, and $\left\langle\sqrt{|\delta v|^{2}}\right\rangle / c_{\mathrm{s}}$ to be $\sim O(\alpha)$ (meaning of order $\alpha$ ), where $c_{\mathrm{s}}$ is the sound speed. We then expand the equations in powers of $\alpha$, and keep only the lowest-order non-vanishing terms in this expansion. The quantities $Q, F$, and $W$ involve products of fluctuating quantities, and are thus $\sim O\left(\alpha^{2}\right)$. We take $R,\left\langle\dot{E}_{\text {source }}\right\rangle, \kappa_{T}, \kappa_{\|}, D_{\mathrm{cr}}, D_{\|}$, and $H_{\text {visc }}$ to be $\sim O\left(\alpha^{2}\right)$, so that, e.g., radiative cooling, conduction, and turbulent heating are of the same order in $\alpha$ in equations (B8) and (B11). (Since the fluctuations are small, we can write, e.g., that $\langle p\rangle \simeq k_{B}\langle\rho\rangle\langle T\rangle / \mu m_{H}$, where $\mu$ is the mean molecular weight.) There are two contributions to the average velocity $\langle v\rangle$. One is driven by the turbulent fluctuations, and, as will be seen below, is of order $\alpha^{2}$. The second arises from the net inflow of mass towards the center of the cluster. In our model, the mass accretion rate is set by the Bondi accretion rate calculated from the plasma parameters at the inner 
radius $r_{1}=0.2 \mathrm{kpc}$, as described in section 2, We treat this second contribution to $\langle v\rangle$ as also of order $\alpha^{2}$.

We now proceed to estimate typical values for $\delta T, \delta \rho$, and $\delta p_{\text {cr }}$ to first order in $\alpha$ - i.e., ignoring terms of order $\alpha^{2}$. Because we take the fluid displacement and the correlation length to be comparable, we need to use a Lagrangian approach to formally integrate the equations. We take the initial position of a fluid element at time $t=0$ to be denoted $r_{0}$, and its position at time $t$ to be

$$
r\left(r_{0}, t\right)=r_{0}+\xi\left(r_{0}, t\right)
$$

where $\xi$ is the displacement of the fluid element. We use the shorthand notation that $\rho(t), T(t)$, $p(t), p_{\mathrm{cr}}(t)$, and $\xi(t)$ are the density, temperature, pressure, cosmic-ray pressure, and displacement at time $t$ of the fluid element that started at position $r_{0}$ at $t=0$. The velocity at time $t$ of the fluid element that starts at $r_{0}$ at $t=0$ is given by

$$
v=\left.\frac{\partial r}{\partial t}\right|_{r_{0}}=\left.\frac{\partial \xi}{\partial t}\right|_{r_{0}} .
$$

We then have that

$$
\left.\frac{\partial}{\partial t}\right|_{r_{0}}=\left.\frac{\partial}{\partial t}\right|_{r}+v \cdot \nabla,
$$

where the spatial derivatives on the right-hand side are with respect to $r$, not $r_{0}$. The Jacobian matrix $\underline{J}$ for the transformation from $r_{0}$ to $r$ is given by the equation

$$
J_{i j}=\frac{\partial r_{i}}{\partial r_{0 j}}
$$

The determinant of this matrix, denoted $J$, satisfies the equation

$$
\left.\frac{\partial}{\partial t} \ln J\right|_{r_{0}}=\nabla \cdot v
$$

where the spatial derivatives on the right-hand side are again with respect to $r$, not $r_{0} 5$ Using equations (B15) and (B17), we rewrite equation (2) as

$$
\left.\frac{\partial \ln \rho}{\partial t}\right|_{r_{0}}=-\left.\frac{\partial \ln J}{\partial t}\right|_{r_{0}} .
$$

\footnotetext{
${ }^{5}$ Equation B17 can be shown as follows. We define $M_{i j}$ to be the determinant of the $2 \times 2$ matrix obtained by deleting the $i^{\text {th }}$ row and $j^{\text {th }}$ column of the matrix $\underline{\underline{J}}$. We can then write that $\partial J / \partial J_{i j}=(-1)^{i+j} M_{i j}$. The inverse of $\underline{\underline{J}}$, which we denote $\underline{\underline{B}}$, satisfies $B_{i j} J_{j k}=\delta_{i k}$, and is given by $B_{i j}=(-1)^{i+j} M_{j i} / J$. We can thus write $\partial J /\left.\partial t\right|_{r_{0}}=$ $\left.\left(\partial J / \partial J_{i j}\right)\left(\partial J_{i j} / \partial t\right)\right|_{r_{0}}=\left.J B_{j i}\left(\partial J_{i j} / \partial t\right)\right|_{r_{0}}$. From the chain rule, $\left(\partial r_{0 i} / \partial r_{j}\right)\left(\partial r_{j} / \partial r_{0 k}\right)=\delta_{i k}$. Thus, $B_{i j}=\partial r_{0 i} / \partial r_{j}$, and $(1 / J) \partial J /\left.\partial t\right|_{r_{0}}=\left(\partial r_{0 j} / \partial r_{i}\right)\left[\left.(\partial / \partial t)\right|_{r_{0}}\left(\partial r_{i} / \partial r_{0 j}\right)\right]=\left(\partial r_{0 j} / \partial r_{i}\right)\left(\partial v_{i} / \partial r_{0 j}\right)=\partial v_{i} / \partial r_{i}$.
} 
We integrate equation ( $(\mathrm{B} 18)$ in time from $t=0$ to $t=\Delta t$ holding $r_{0}$ fixed (as in the time integrals below), where

$$
\Delta t=\frac{l}{u_{\mathrm{L}}}
$$

is the "mixing time," and $u_{L}$ is the rms radial velocity in the local mixing length theory. We set $\xi(0)=0$ and $\rho(0)=\langle\rho\rangle$, where $\langle\rho\rangle$ is shorthand notation for the average density at $r_{0}$. We then obtain

$$
\frac{\rho(\Delta t)}{\langle\rho\rangle}=\frac{1}{J(\Delta t)}
$$

where $J(\Delta t)$ is the Jacobian at $t=\Delta t$ evaluated for the initial position $r_{0}$. If we start at $t=0$ with a fluid element of infinitesimal volume $d^{3} r_{0}$ centered at $r_{0}$, then at time $\Delta t$ its volume is $d^{3} r=$ $J(\Delta t) d^{3} r_{0}$. Thus, equation ( $(\underline{\mathrm{B} 20})$ is a statement of mass conservation. The Lagrangian density perturbation at time $\Delta t$ is

$$
\Delta \rho_{\text {Lag }} \equiv \rho(\Delta t)-\rho(0)=\rho(\Delta t)-\langle\rho\rangle .
$$

Combining equations ( $(\mathrm{B} 20)$ and ( $(\overline{B 21})$, we write

$$
\frac{\Delta \rho_{\mathrm{Lag}}}{\langle\rho\rangle}=\frac{1}{J(\Delta t)}-1 \text {. }
$$

To solve for the pressure fluctuation, we write equation (4) in the form

$$
\left.\frac{\partial p}{\partial t}\right|_{r_{0}}=-\left.\gamma p \frac{\partial \ln J}{\partial t}\right|_{r_{0}}+(\gamma-1) \delta H_{\mathrm{tc}}
$$

where

$$
H_{\mathrm{tc}}=\nabla \cdot\left(\kappa_{\|} \hat{b} \hat{b} \cdot \nabla T\right)
$$

is the rate of heating due to thermal conduction, and $\delta H_{\mathrm{tc}}$ is the deviation of $H_{\mathrm{tc}}$ from its average value. In writing equation ( $(\underline{\mathrm{B} 23})$, we have dropped terms of order $\alpha^{2}$. [ $\delta H_{\mathrm{tc}} \sim O(\alpha)$ since $\delta T \sim$ $O(\alpha), \kappa_{\|} \sim O\left(\alpha^{2}\right)$, and $\nabla^{2} \delta T \sim \delta T / l^{2} \sim O\left(\alpha^{-1}\right)$.] We integrate equation (B23) from $t=0$ to $t=\Delta t$, setting $\xi(0)=0$ and $p(0)=\langle p\rangle$, where $\langle p\rangle$ is the average density at $r_{0}$. To first order in $\alpha$, we can replace $\gamma p \partial \ln J /\left.\partial t\right|_{r_{0}}$ with $\gamma\langle p\rangle \partial \ln J /\left.\partial t\right|_{r_{0}}$. We thus obtain

$$
\Delta p_{\text {lag }}=-\gamma\langle p\rangle \ln J(\Delta t)+(\gamma-1) \int_{0}^{\Delta t} \delta H_{\mathrm{tc}}(t) d t
$$

where

$$
\Delta p_{\text {Lag }} \equiv p(\Delta t)-p(0)=p(\Delta t)-\langle p\rangle,
$$

is the Lagrangian pressure perturbation at time $\Delta t$. Because the density fluctuations are small, the value of $J(\Delta t)$ is very close to 1 . Writing $J(\Delta t)=1+x$, the quantity $x$ is of order $\alpha$. Thus, $\ln J(\Delta t)=x+O\left(\alpha^{2}\right)=1-[J(\Delta t)]^{-1}+O\left(\alpha^{2}\right)$ and

$$
\ln J(\Delta t)=-\frac{\Delta \rho_{\mathrm{Lag}}}{\langle\rho\rangle}+O\left(\alpha^{2}\right) .
$$


To first order in $\alpha$, we can thus rewrite equation (B25) as

$$
\Delta p_{\text {lag }}=\frac{\gamma\langle p\rangle \Delta \rho_{\mathrm{Lag}}}{\langle\rho\rangle}+(\gamma-1) \int_{0}^{\Delta t} \delta H_{\mathrm{tc}}(t) d t .
$$

We have been analyzing a fluid element that starts off at $t=0$ as an "average" fluid element, and we thus take $\delta T=0$ and $\delta H_{\mathrm{tc}}=0$ at $t=0$. When this fluid element is displaced radially outwards a distance $l$, it remains magnetically connected to the same set of fluid elements to which it was initially connected (at least until it is mixed into the surrounding fluid, at which point it is assumed that the magnetic field in the fluid parcel is randomized). This is depicted schematically in figure 7. If its temperature remains unchanged as it moves [i.e., if $\delta T_{\text {Lag }} \equiv T(t)-T(0)=0$ ], then the effects of thermal conduction are unchanged and $\delta H_{\mathrm{tc}}$ will remain zero. However, if its temperature decreases, then more heat will be conducted into the fluid element, and $\delta H_{\mathrm{tc}}$ will increase. Thermal conduction will thus act to restore the temperature to its initial value (i.e. to keep $\delta T_{\text {Lag }}=0$ ), with $\delta H_{\mathrm{tc}} \propto-\delta T_{\text {Lag }}$ for small values of $\delta T_{\mathrm{Lag}}$. To estimate $\delta H_{\mathrm{tc}}$, we note that the mixing length $l$ is comparable to the correlation lengths of both the convective turbulence and the temperature fluctuations. Thus, $\left|\nabla \delta T_{\mathrm{Lag}}\right| \sim \delta T_{\mathrm{Lag}} / l$, and we have the order-of-magnitude relation

$$
\delta H_{\mathrm{tc}} \sim-\frac{\kappa_{\|} \delta T_{\mathrm{Lag}}}{l_{T}^{2}} .
$$

We estimate that

$$
(\gamma-1) \int_{0}^{\Delta t} \delta H_{\mathrm{tc}} d t=-\frac{0.3(\gamma-1) \kappa_{\|} \Delta T_{\mathrm{Lag}} \Delta t}{l^{2}}
$$

where $\Delta T_{\mathrm{Lag}}=\delta T_{\mathrm{Lag}}(\Delta t)$ is the Lagrangian temperature perturbation at time $\Delta t$, and the numerical factor of 0.3 is chosen somewhat arbitrarily to reflect (1) our expectation that the length scale of the temperature fluctuations is somewhat larger than $l$, which is just the radial component of the displacement vector, not the full modulus of $\xi$, and (2) the fact that $\delta H_{\text {tc }}$ increases from zero to its maximum value as $t$ ranges from 0 to $\Delta t$, so the typical value of $\delta H_{\text {tc }}$ is less than its value at $t=\Delta t$. Finally, we obtain the relation

$$
\Delta p_{\text {lag }}=\frac{\gamma\langle p\rangle \Delta \rho_{\mathrm{Lag}}}{\langle\rho\rangle}-\frac{0.3(\gamma-1) \kappa_{\|} \Delta T_{\mathrm{Lag}} \Delta t}{l^{2}}
$$

To first order in $\alpha$,

$$
\frac{\Delta T_{\mathrm{Lag}}}{\langle T\rangle}=\frac{\Delta p_{\mathrm{Lag}}}{\langle p\rangle}-\frac{\Delta \rho_{\mathrm{Lag}}}{\langle\rho\rangle},
$$

where $\langle T\rangle$ is the average temperature at $r_{0}$. Equations $(\underline{\mathrm{B} 31})$ and $(\mathrm{B} 32)$ combine to give

$$
\Delta p_{\text {Lag }}=\Delta \rho_{\text {Lag }} \frac{\langle p\rangle}{\langle\rho\rangle}\left(\frac{\gamma+a_{1}}{1+a_{1}}\right),
$$




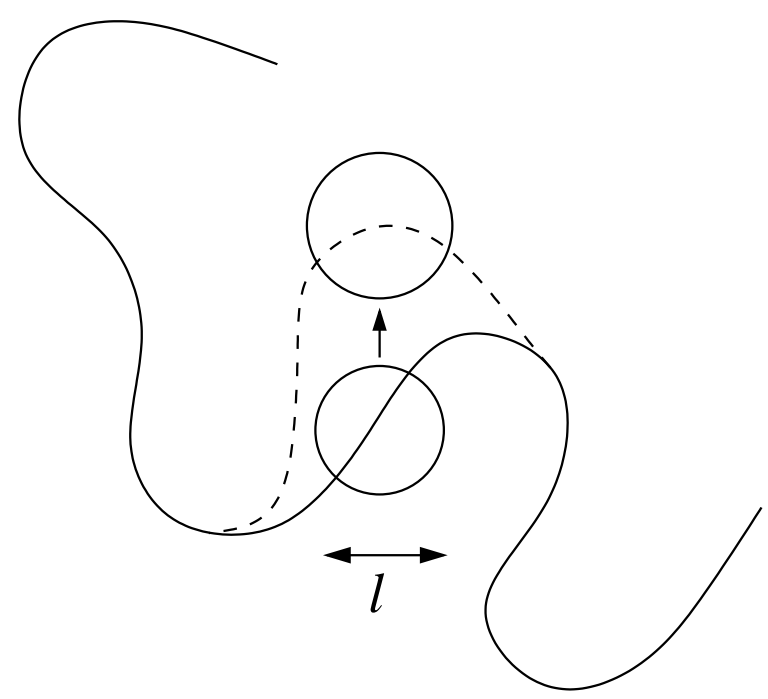

Fig. 7.- Schematic diagram of a rising fluid parcel. The solid line is a magnetic field line passing through the parcel's initial location. The dashed line is an idealization of how the field line changes as a result of the fluid parcel's displacement.

where

$$
a_{1}=\frac{0.3(\gamma-1) \kappa_{\|}\langle T\rangle}{l u_{\mathrm{L}}\langle p\rangle}
$$

is roughly the ratio of the mixing time $\Delta t$ to the time for heat to diffuse a distance $l$ along the magnetic field. When $a_{1} \ll 1$ the thermal plasma expands adiabatically, and when $a_{1} \gg 1$ the thermal plasma expands isothermally.

To solve for the cosmic-ray pressure fluctuation, we write equation (5) in the form

$$
\left.\frac{\partial p_{\mathrm{cr}}}{\partial t}\right|_{r_{0}}=-\left.\gamma_{\mathrm{cr}} p_{\mathrm{cr}} \frac{\partial \ln J}{\partial t}\right|_{r_{0}}+\delta H_{\mathrm{diff}}+\left(\gamma_{\mathrm{cr}}-1\right) \delta \dot{E}_{\mathrm{source}}
$$

where

$$
H_{\text {diff }}=\nabla \cdot\left(D_{\|} \hat{b} \hat{b} \cdot \nabla p_{\mathrm{cr}}\right)
$$

$\delta H_{\text {diff }}$ is the deviation of $H_{\text {diff }}$ from its average value, $\dot{E}_{\text {source }}$ is the cosmic-ray energy per unit volume generated by the central radio source, and $\delta \dot{E}_{\text {source }}$ is the deviation of $\dot{E}_{\text {source }}$ from its average value. As discussed in section 2.7, $\delta \dot{E}_{\text {source }}$ can be significantly larger than $\left\langle\dot{E}_{\text {source }}\right\rangle$ if the cosmic rays are accelerated in a small fraction of the volume. We thus treat $\delta \dot{E}_{\text {source }}$ as $O(\alpha)$ and $\left\langle\dot{E}_{\text {source }}\right\rangle$ as $O\left(\alpha^{2}\right)$. We integrate equation (B35) from $t=0$ to $t=\Delta t$, setting $\xi(0)=0$ and $p_{\mathrm{cr}}(0)=\left\langle p_{\mathrm{cr}}\right\rangle$, where $\left\langle p_{\mathrm{cr}}\right\rangle$ is the average cosmic-ray pressure at $r_{0}$. To first order in $\alpha$, we can replace $\gamma_{\mathrm{cr}} p_{\mathrm{cr}} \partial \ln J /\left.\partial t\right|_{r_{0}}$ with $\gamma_{\mathrm{cr}}\left\langle p_{\mathrm{cr}}\right\rangle \partial \ln J /\left.\partial t\right|_{r_{0}}$. Using equation (B), we obtain

$$
\Delta p_{\mathrm{cr}, \mathrm{Lag}}=\frac{\gamma_{\mathrm{cr}}\left\langle p_{\mathrm{cr}}\right\rangle \Delta \rho_{\mathrm{Lag}}}{\langle\rho\rangle}+\int_{0}^{\Delta t}\left[\delta H_{\mathrm{diff}}+\left(\gamma_{\mathrm{cr}}-1\right) \delta \dot{E}_{\text {source }}\right] d t
$$


where

$$
\Delta p_{\text {cr, Lag }}=p_{\text {cr }}(\Delta t)-\left\langle p_{\text {cr }}\right\rangle,
$$

is the Lagrangian cosmic-ray pressure perturbation at time $\Delta t$.

We treat parallel cosmic-ray diffusion in the same way as parallel thermal conduction and make the estimate

$$
\int_{0}^{\Delta t} \delta H_{\text {diff }} d t=-\frac{0.3 D_{\|} \Delta p_{\mathrm{cr}, \mathrm{Lag}} \Delta t}{l^{2}} .
$$

We assume that $\delta \dot{E}_{\text {source }}$ is typically positive in outwardly moving fluid elements and negative in inwardly moving fluid elements. Here, we are focusing on a a fluid element that is moving outwards (the discussion can be repeated with little alteration for inwardly moving fluid parcels), and thus we treat $\delta \dot{E}_{\text {source }}$ as positive. We then make the estimate that

$$
\int_{0}^{\Delta t} \delta \dot{E}_{\text {source }} d t=\delta \dot{E}_{\mathrm{rms}} \Delta t
$$

where $\delta \dot{E}_{\text {rms }}$ is the rms value of $\delta \dot{E}_{\text {source }}$, which is determined from equation (35). Substituting (B39), and (B40) into equation (B37), we obtain

$$
\Delta p_{\mathrm{cr}, \mathrm{Lag}}=\frac{\gamma_{\mathrm{cr}}\left\langle p_{\mathrm{cr}}\right\rangle \Delta \rho_{\mathrm{Lag}}}{\left(1+a_{2}\right)\langle\rho\rangle}+\left(\gamma_{\mathrm{cr}}-1\right) \delta \dot{E}_{\mathrm{rms}} \tau,
$$

where

$$
\tau=\left(\frac{u_{L}}{l}+\frac{0.3 D_{\|}}{l^{2}}\right)^{-1}
$$

is the effective time during which cosmic rays can accumulate in the fluid parcel as a result of the cosmic-ray source term. ( $\tau$ is roughly the shorter of the mixing time $l / u_{L}$ and the diffusion time $l^{2} / D_{\|}$.) The quantity $a_{2}$ is given by

$$
a_{2}=\frac{0.3 D_{\|}}{l u_{\mathrm{L}}}
$$

and is approximately the ratio of $\Delta t$ to the time for cosmic rays to diffuse a distance $l$ along the magnetic field. When $a_{2} \ll 1$, the cosmic rays expand adiabatically if $\delta \dot{E}_{\mathrm{rms}}=0$. When $a_{2} \gg 1$ the cosmic ray pressure in the fluid element remains constant as the element is displaced if $\delta \dot{E}_{\text {rms }}=0$, as in the linear Parker instability in the large- $D_{\|}$limit (Parker 1966, Shu 1974, Ryu et al 2003).

Since it is assumed that the convection is subsonic, the total pressure in the fluid element remains approximately the same as the average total pressure in the fluid element's surroundings. We take

$$
\hat{r} \cdot \xi(\Delta t)=l,
$$

and thus to first order in $\alpha$

$$
\Delta p_{\text {Lag }}+\Delta p_{\text {cr,Lag }}=l \frac{d}{d r}\left\langle p_{\text {tot }}\right\rangle .
$$


Adding equations ( $(\underline{B 33})$ and $(\underline{B} 41)$ and using equation $(\underline{B} 45)$, we obtain

$$
l \frac{d}{d r}\left\langle p_{\mathrm{tot}}\right\rangle=c_{\mathrm{eff}}^{2} \Delta \rho_{\mathrm{Lag}}+\left(\gamma_{\mathrm{cr}}-1\right) \delta \dot{E}_{\mathrm{rms}} \tau,
$$

where

$$
c_{\text {eff }}=\left[\left(\frac{\gamma+a_{1}}{1+a_{1}}\right) \frac{\langle p\rangle}{\langle\rho\rangle}+\frac{\gamma_{\mathrm{cr}}\left\langle p_{\mathrm{cr}}\right\rangle}{\left(1+a_{2}\right)\langle\rho\rangle}\right]^{1 / 2}
$$

is an effective sound speed for the medium.

The fluctuating quantities appearing in equations ( $\mathrm{B} 5)$, (B9), and (B10) are Eulerian fluctuations, in that they involve the difference between some quantity and the average of that quantity at the same location. To first order in $\alpha$, we can write the Eulerian density fluctuation of our outwardly displaced fluid element at time $\Delta t$, denoted $\Delta \rho$, as

$$
\Delta \rho=\Delta \rho_{\text {Lag }}-l \frac{d\langle\rho\rangle}{d r} .
$$

Combining equations ( $(\mathrm{B} 46)$ and $(\underline{\mathrm{B} 48})$, we find that

$$
\Delta \rho=l\left(\frac{1}{c_{\mathrm{eff}}^{2}} \frac{d\left\langle p_{\mathrm{tot}}\right\rangle}{d r}-\frac{d\langle\rho\rangle}{d r}\right)-\frac{\left(\gamma_{\mathrm{cr}}-1\right) \delta \dot{E}_{\mathrm{rms}} \tau}{c_{\mathrm{eff}}^{2}} .
$$

The fluid is convectively stable if an outwardly displaced parcel is heavier than its surroundings (i.e., if $\Delta \rho>0$ ) for any value of $u_{L}$. We note that as $u_{L}$ increases, $a_{1}$ and $a_{2}$ decrease, $c_{\text {eff }}^{2}$ increases, and $\tau$ decreases. Since $d\left\langle p_{\text {tot }}\right\rangle / d r<0$, it follows that

$$
\frac{d}{d u_{L}} \Delta \rho>0
$$

Thus, if $\Delta \rho>0$ as $u_{L} \rightarrow 0$, then $\Delta \rho>0$ for any $u_{L}$ ( $u_{L}$ is by definition non-negative), and the medium is convectively stable. On the other hand, if $\Delta \rho<0$ as $u_{L} \rightarrow 0$, then the medium is convectively unstable. The necessary and sufficient condition for convective stability is thus that $\Delta \rho$ be positive in the limit $u_{L} \rightarrow 0$. As $u_{L} \rightarrow 0$, we have that $a_{1} \rightarrow \infty, a_{2} \rightarrow \infty, \tau \rightarrow l^{2} /\left(0.3 D_{\|}\right)$, and $c_{\text {eff }}^{2} \rightarrow\langle p\rangle /\langle\rho\rangle$. For constant mean molecular weight $\mu$, this then leads to the stability criterion

$$
l\left(n k_{B} \frac{d T}{d r}+\frac{d p_{\mathrm{cr}}}{d r}\right)-\frac{\left(\gamma_{\mathrm{cr}}-1\right) \delta \dot{E}_{\mathrm{rms}} l^{2}}{0.3 D_{\|}}>0,
$$

where $n=\rho /\left(\mu m_{H}\right)$ is the number density of thermal particles. If one sets $\delta \dot{E}_{\mathrm{rms}}$ to zero, then equation (B51) reduces to the stability criterion derived by Chandran (2005) and Chandran \& Dennis (2006). Here, we have kept the fluctuations in $\dot{E}_{\text {source, }}$, which act to destabilize the medium to convection, since localized excesses in the cosmic-ray pressure lead to pockets of buoyant, lower-density fluid. 
If the convective stability criterion is satisfied at some radius, we set the local convective velocity $u_{\mathrm{L}}$ to zero at that radius. Otherwise the fluid is convectively unstable, and we estimate the value of $u_{\mathrm{L}}$ by solving the polynomial equation

$$
\frac{\langle\rho\rangle u_{\mathrm{L}}^{2}}{2}=\left|\frac{l \Delta \rho}{16} \frac{d \Phi}{d r}\right| .
$$

Equation (B52) states that the mean radial kinetic energy of the fluid element is the mixing length times the buoyancy force on the fully displaced parcel times the numerical factor of $1 / 16$ that is commonly used in one-fluid mixing length theory (Cox \& Giuli 1968). Once $u_{\mathrm{L}}$ is found, we determine $\Delta \rho_{\text {Lag }}$ and $\Delta p_{\text {Lag }}$ using equations ( $(\mathrm{B} 46)$ and (B33), respectively. The Eulerian pressure perturbation at time $\Delta t$, denoted $\Delta p$, is then given by the equation

$$
\Delta p=\Delta p_{\mathrm{Lag}}-l \frac{d\langle p\rangle}{d r} .
$$

We then estimate the quantity $F$ in equation $(\mathrm{B} 9)$ to be

$$
F_{\mathrm{L}}=\frac{c_{\mathrm{avg}} u_{\mathrm{L}} \Delta p}{\gamma-1}
$$

Here, as below, the L subscript is used to denote the estimate obtained using local mixing length theory. We set

$$
c_{\text {avg }}=1 / 2
$$

to match standard treatments of local one-fluid mixing length theory (Cox \& Giuli 1968). We estimate the quantity $Q$ in equation (B5) to be

$$
Q_{L}=c_{\mathrm{avg}} u_{L} \Delta \rho,
$$

with $\Delta \rho$ determined from equation (B49). We estimate the quantity $W$ in equation (B10) by noting that $\int_{0}^{\Delta t} \nabla \cdot v d t=-\ln \left[\frac{\rho(\Delta t)}{\langle\rho\rangle}\right]=-\ln \left[1+\frac{\Delta \rho_{\text {Lag }}}{\langle\rho\rangle}\right] \simeq-\frac{\Delta \rho_{\text {Lag }}}{\langle\rho\rangle}$. Thus, the typical value of $\nabla \cdot \delta v$ is $\frac{1}{\Delta t}\left(-\frac{\Delta \rho_{\text {Lag }}}{\langle\rho\rangle}\right)=-\frac{u \Delta \rho_{\text {Lag }}}{l\langle\rho\rangle}$. We thus set

$$
W_{\mathrm{L}}=-\frac{c_{\mathrm{avg}} u_{L} \Delta p \Delta \rho_{\mathrm{Lag}}}{l\langle\rho\rangle}
$$

Having estimated $Q, F$, and $W$ using local mixing length theory, we now use these estimates as the basis for a nonlocal theory. In the study of Ulrich (1976), the nonlocal heat flux is given by a weighted spatial average of the heat flux obtained from local mixing length theory. We adopt the same approach and set

$$
F_{\mathrm{NL}}(z)=\int_{-\infty}^{\infty} d z_{1} F_{\mathrm{L}}\left(z_{1}\right) \psi_{F}\left(z-z_{1}\right)
$$




$$
W_{\mathrm{NL}}(z)=\int_{-\infty}^{\infty} d z_{1} W_{\mathrm{L}}\left(z_{1}\right) \psi_{W}\left(z-z_{1}\right)
$$

and

$$
Q_{\mathrm{NL}}(z)=\int_{-\infty}^{\infty} d z_{1} Q_{\mathrm{L}}\left(z_{1}\right) \psi_{Q}\left(z-z_{1}\right)
$$

where

$$
z=\ln \left(\frac{r}{r_{\text {ref }}}\right)
$$

$r_{\text {ref }}$ is an unimportant constant, and the NL subscripts denote values in our nonlocal theory. Different forms for the kernel function $\psi_{F}$ were considered by Ulrich (1976). Here, we adopt the following values:

$$
\psi_{Q}(x)=\psi_{F}(x)= \begin{cases}\alpha^{-1} e^{-x / \alpha} & \text { if } x>0 \\ 0 & \text { if } x \leq 0\end{cases}
$$

and

$$
\psi_{W}(x)=\left\{\begin{array}{ll}
\alpha_{W}^{-1} e^{-x / \alpha_{W}} & \text { if } x>0 \\
0 & \text { if } x \leq 0
\end{array} .\right.
$$

Equations ( $(\overline{\mathrm{B} 58})$ through $(\underline{\mathrm{B} 63})$ are equivalent to the differential equations

$$
\begin{aligned}
& \alpha r \frac{d F_{\mathrm{NL}}}{d r}+F_{\mathrm{NL}}=F_{\mathrm{L}}, \\
& \alpha r \frac{Q_{\mathrm{NL}}}{d r}+Q_{\mathrm{NL}}=Q_{\mathrm{L}},
\end{aligned}
$$

and

$$
\alpha_{W} r \frac{W_{\mathrm{NL}}}{d r}+W_{\mathrm{NL}}=W_{\mathrm{L}} .
$$

For $r>r_{\text {conv }}$, where $r_{\text {conv }}$ is the largest radius at which the fluid is locally unstable to convection, $F_{L}=0$ and $F_{\mathrm{NL}} \propto r^{-1 / \alpha}$. When $F$ and $W$ are set equal to $F_{\mathrm{NL}}$ and $W_{\mathrm{NL}}$ in equations (B8) and (B11), the terms containing $F_{\mathrm{NL}}$ are $\propto r^{-1-1 / \alpha}$ for $r>r_{\text {conv }}$. To obtain the same scaling for the terms containing $W_{\mathrm{NL}}$, the value of $\alpha_{W}$ is determined from the equation

$$
\alpha_{W}^{-1}=\alpha^{-1}+1
$$

Equations (B62) and (B63) represent a one-sided average, in the sense that the nonlocal quantities $F_{\mathrm{NL}}, Q_{\mathrm{NL}}$, and $W_{\mathrm{NL}}$ depend only on the values of $F_{L}, Q_{L}$, and $W_{L}$ at smaller radii. A more sophisticated nonlocal theory could be developed along different lines (see e.g. Travis \& Matsushima 1973, Ulrich 1976, Xiong 1991, Grossman, Narayan, \& Arnett 1993), but is beyond the scope of this paper.

The final equations for our mixing-length model are then equations $(\overline{\mathrm{B} 4}),(\overline{\mathrm{B} 6}),(\overline{\mathrm{B} 8}),(\overline{\mathrm{B} 11})$, ( $(\mathrm{B} 64),(\mathrm{B} 65)$, and (B66), which form a system of two second-order ordinary differential equations 
(ODEs), four first-order ODEs, and one algebraic equation [equation (B4)] for the seven variables $\langle\rho\rangle,\left\langle v_{r}\right\rangle\langle T\rangle,\left\langle p_{\mathrm{cr}}\right\rangle, F_{\mathrm{NL}}, Q_{\mathrm{NL}}$, and $W_{\mathrm{NL}}$. Eight boundary conditions are required to specify a solution. Two boundary conditions are obtained by requiring that the model density and temperature match the observed values $\rho_{\text {outer }}$ and $T_{\text {outer }}$ at the outer radius $r_{\text {outer }}$. For seven of the eight clusters in our sample (all except Virgo), we choose $r_{\text {outer }}$ to be the center of the first radial bin outside the cooling radius $r_{\text {cool }}$. Values of $r_{\text {cool }}$ for each cluster are given in table 2, For Virgo, we take $r_{\text {outer }}$ to be the outermost data point, which lies inside of $r_{\text {cool }}$. Since we do not solve all the way in to the sonic point, we are forced to pick inner boundary conditions (at $r_{1}=0.2 \mathrm{kpc}$ ) in a somewhat arbitrary way. We take $d\langle T\rangle / d r, d\left\langle p_{\mathrm{cr}}\right\rangle / d r, F_{\mathrm{NL}}, W_{\mathrm{NL}}$, and $Q_{\mathrm{NL}}$ to vanish at $r=r_{1}$. These "no-flux" boundary conditions set the diffusive and turbulent energy fluxes to zero at the inner boundary. Although this choice is undoubtedly inaccurate, we expect that it has only a small effect on our solution for the structure of the intracluster medium at $r \gg r_{1}$. The eighth boundary condition is obtained by assuming that $\left\langle p_{\mathrm{cr}}\right\rangle \rightarrow 0$ as $r \rightarrow \infty$. This condition is translated into a condition on $\left\langle p_{\mathrm{cr}}\right\rangle$ at $r_{\text {outer }}$ as follows. The value of $r_{\text {outer }}$ is chosen to be significantly greater than $r_{\text {source }}$ and much greater than $D_{0} / v_{d}$, so that for $r>r_{\text {outer }}, \dot{E}_{\text {source }}$ is negligible and $D_{\text {cr }} \simeq v_{d} r 6$ In addition, $r_{\text {outer }}$ is taken to lie outside $r_{\text {conv }}$, the largest radius at which the intracluster medium is locally convectively unstable, so that $F_{\mathrm{NL}}=F_{\text {outer }}\left(r / r_{\text {outer }}\right)^{-1 / \alpha}$ and $W_{\mathrm{NL}}=W_{\text {outer }}\left(r / r_{\text {outer }}\right)^{-1-1 / \alpha}$ for $r>r_{\text {outer }}$, where $F_{\text {outer }}$ and $W_{\text {outer }}$ are the values of $F_{\mathrm{NL}}$ and $W_{\mathrm{NL}}$ at $r=r_{\text {outer }}$. We also take $\left\langle v_{r}\right\rangle$ to be negligible for $r>r_{\text {outer }}$. This latter assumption is reasonable, since $Q_{\mathrm{NL}}=Q_{\text {outer }}\left(r / r_{\text {outer }}\right)^{-1 / \alpha}$ for $r>r_{\text {conv }}$, where $Q_{\text {outer }}$ is the value of $Q_{\mathrm{NL}}$ at $r_{\text {outer }}$. The resulting value of $\left\langle v_{r}\right\rangle$ is significantly less than $v_{d}$ for $r>r_{\text {conv }}$ in the numerical solutions we present in section 3 , and thus $\left\langle v_{r}\right\rangle$ plays only a small role in equation $(\underline{\mathrm{B} 8})$ at $r>r_{\mathrm{conv}}$. Solving equation $(\underline{\mathrm{B} 8})$ and requiring that $\left\langle p_{\mathrm{cr}}\right\rangle \rightarrow 0$ as $r \rightarrow \infty$, we find that for $r \geq r_{\text {outer }}$

$$
\frac{d\left\langle p_{\mathrm{cr}}\right\rangle}{d r}=\frac{\chi}{v_{d} r^{1+1 / \alpha}}-\frac{2\left\langle p_{\mathrm{cr}}\right\rangle}{r}
$$

where

$$
\chi=(2 \alpha-1)(\gamma-1) F_{\text {outer }} r_{\text {outer }}^{1 / \alpha}+\alpha\left(\gamma_{\text {cr }}-1\right) W_{\text {outer }} r_{\text {outer }}^{1+1 / \alpha} .
$$

Equation (B68) applied at $r=r_{\text {outer }}$ provides the eighth boundary condition. We then solve our system of equations using a shooting method. We guess the values of $\langle\rho\rangle,\langle T\rangle$, and $\left\langle p_{\mathrm{cr}}\right\rangle$ at $r=r_{1}$ and then integrate the equations from $r=r_{1}$ to $r=r_{\text {outer }}$. We then update our three guesses using Newton's method until the three boundary conditions at $r_{\text {outer }}$ are met.

To compare to future observations and to analyze the turbulent diffusion of metals in the ICM [see, e.g., Rebusco et al (2005)], it is of interest to calculate the rms turbulent velocity. We define

\footnotetext{
${ }^{6}$ The case $v_{d}=0$ requires a different approach and is not treated in this paper.
} 
a nonlocal turbulent velocity, $u_{\mathrm{NL}}$, through the equation

$$
\alpha r \frac{d u_{\mathrm{NL}}}{d r}+u_{\mathrm{NL}}=u_{\mathrm{L}}
$$

Since $u_{L}$ (and thus $u_{\mathrm{NL}}$ ) is an estimate of the radial component of the velocity of a convective fluid element, the full rms turbulent velocity is roughly

$$
u_{\mathrm{rms}}=\sqrt{3} u_{\mathrm{NL}}
$$

which is the quantity plotted in figure 5

\section{REFERENCES}

Allen, S. W., Dunn, R. J. H., Fabian, A. C., Taylor, G. B., Reynolds, C. S. 2006, MNRAS, 372, 21

Balbus, S. 2000, ApJ, 534, 420

Balbus, S. 2001, ApJ, 562, 909

Begelman, M. C. 2001, in ASP Conf. Proc., 240, Gas and Galaxy Evolution, ed. J. E. Hibbard, M. P. Rupen, \& J. H. van Gorkom (San Fransisco: ASP), 363

Begelman, M. C. 2002, in ASP Conf. Proc., 250, Particles and Fields in Radio Galaxies, ed. R. A. Laing, \& K. M. Blundell (San Fransisco: ASP), 443

Bieber, J. Matthaeus, W., Smith, C., Wanner, W., Kallenrode, M., \& Wibberenz, G. 1994, ApJ, 420, 294

Binney, J., \& Tabor, G. 1995, MNRAS, 276, 663

Birzan, L., Rafferty, D., McNamara, B., Wise, M., \& Nulsen, P. 2004, ApJ, 607, 800

Blanton, E., Sarazin, C., \& McNamara, B. 2003, ApJ, 585, 227

Böhringer, H. et al 2001, A\&A, 365, L181

Böhringer, H., \& Morfill, G. 1988, ApJ, 330, 609

Böhringer, H., Matsushita, K., Churazov, E., \& Finoguenov, A. 2004a, in The Riddle of Cooling Flows and Clusters of Galaxies, ed. Reiprich, T., Kempner, J., \& Soker, N., E3,

\section{http://www.astro.virginia.edu/coolflow/proc.php}

Bondi, H. 1952, MNRAS, 112, 159

Braginskii, S. I. 1965, in Reviews of Plasma Physics, vol. 1, ed. M. A. Leontovich (New York: Consultants Bureau), 205

Cattaneo, A., \& Teyssier, R. 2007, MNRAS, 192 
Chandran, B. 2000a, Phys. Rev. Lett., 85, 4656

Chandran, B. 2004, ApJ, 616, 169 (Paper I)

Chandran, B. 2005, ApJ, 632, 809 (Paper II)

Chandran, B., \& Cowley, S. 1998, Phys. Rev. Lett., 80, 3077

Chandran, B., \& Dennis, T. 2006, ApJ, 642, 140

Chandran, B., Maron, J. 2004, ApJ, 602, 170

Churazov, E., Brüggen, M., Kaiser, C., Böhringer, H., \& Forman, W. 2001, ApJ, 554, 261

Churazov, E., Forman, W., Jones, C., Sunyaev, R., \& Böhringer, H. 2004, MNRAS, 347, 29

Churazov, E., Sunyaev, R., Forman, W., \& Böhringer, H. 2002, MNRAS, 332, 729

Ciotti, L., \& Ostriker, J. 1997, ApJ, 487, L105

Ciotti, L., \& Ostriker, J. 2001, ApJ, 551, 131

Ciotti, L., \& Ostriker, J., \& Pellegrini, S. 2004, in Plasmas in the Laboratory and in the Universe: New Insights and New Challenges,

Cox, D. P. 2000, Allen's Astrophysical Quantities (New York: AIP)

David, L. P., Nulsen, P. E. J., McNamara, B. R., Forman, W., Jones, C., Ponman, T., Robertson, B., Wise, M. 2001, ApJ, 557, 546

Dennis, T., \& Chandran, B. 2005, 622, 205

Di Matteo, T., Allen, S. W., Fabian, A. C., Wilson, A. S., \& Young, A. J. 2003, ApJ, 582, 133

Drury, L., \& Volk, H. 1981, ApJ, 248, 344

Eilek, J. 2004, in The Riddle of Cooling Flows and Clusters of Galaxies, ed. Reiprich, T., Kempner, J., \& Soker, N., E13, http://www.astro.virginia.edu/coolflow/proc.php

Fabian, A. C. 1994, Ann. Rev. Astr. Astrophys., 32, 277

Fabian, A. C., Sanders, J., Allen, S., Crawford, C., Iwasawa, K., Johnstone, M., Schmidt, R., \& Taylor, G. 2003, MNRAS, 344, L43

Fabian, A. C. 1994, Ann. Rev. Astr. Astrophys., 32, 277

Goldreich, P. \& Sridhar, S. 1995, ApJ, 438, 763

Graham, A., Merritt, D., Moore, B., Diemand, J., \& Terzić, B. 2006, ApJ, 132, 2711

Graham, A., Lauer, T. R., Colless, M., \& Postman, M. 1996, ApJ, 465, 534

Grossman, S., Narayan, R., \& Arnett, D. 1993, ApJ, 407, 284

Hernquist, L. 1990, ApJ, 356, 359 
Hoeft, M., \& Brüggen, M. 2004, ApJ, 617, 896

Kaastra, J. S., Tamura, T., Peterson, J., Bleeker, J., Ferrigno, C., Kahn, S., Paerels, F., Piffaretti, R., Branduardi-Raymont, G., \& Böhringer, H. 2004, A\&A, 413, 415

Kronberg, P. 1994, Rep. Prog. Phys., 57, 325

Jones, T., \& Kang, H. 1990, ApJ, 363, 499

Kim, W., \& Narayan, R. 2003, ApJ, 596, L139

Lauer, T. R., Faber, S. M., Richstone, D., Gebhardt, K., Tremaine, S., Postman, M., Dressler, A., Aller, M. C., Filippenko, A. V., Green, R., Ho, L. C., Kormendy, J., Magorrian, J., Pinkney, J. 2007, ApJ, 662, 808

Lazarian, A. 2006, ApJL, 645, 25

Lewis, G. F., Babul, A., Katz, N., Quinn, T., Hernquist, L., \& Weinberg, D. 2000, ApJ, 536, 623

Loewenstein, M., \& Fabian, A. 1990, MNRAS, 242120

Loewenstein, M., Zweibel, E., \& Begelman, M. 1991, ApJ, 377, 392

Maron, J., Chandran, B., \& Blackman, E. 2004, Phys. Rev. Lett., 92, id. 045001

McLaughlin, D. 1999, ApJ, 512, L9

Molendi, S., \& Pizzolatao, F. 2001, ApJ, 560, 194

Nagai, D., \& Kravtsov, A. 2004, in IAU Colloq. 195, Outskirts of Galaxy Clusters: Intense Life in the Suburbs, ed. A. Diaferio (Cambridge: Cambridge Univ. Press), 296

Narayan, R., \& Medvedev, M. 2001, ApJ, 562, 129

Navarro, J., Frenk, C., \& White, S. 1997, ApJ, 490, 493

Nulsen, P. 2004, in The Riddle of Cooling Flows and Clusters of Galaxies, ed. Reiprich, T., Kempner, J., \& Soker, N., E30, http://www.astro.virginia.edu/coolflow/proc.php

Owen, F., \& Ledlow, M. 1997, ApJS, 108, 410

Parrish, I., \& Stone, J., 2005, ApJ, 633, 334

Parrish, I., \& Stone, J. 2006, astro-ph/0612195

Paturel, G.; Petit, C.; Prugniel, Ph.; Theureau, G.; Rousseau, J.; Brouty, M.; Dubois, P.; Cambrsy, L. 2003, A\&A, 412, 45-55

Peterson, J. R., et al 2001, A\&A, 365, L104

Peterson, J. R., Kahn, S., Paerels, F., Kaastra, J., Tamura, T., Bleeker, J., Ferrigo, C., \& Jernigan, J. 2003, ApJ, 590, 207

Piffaretti, R., Jetzer, Ph., Kaastra, J., Tamura, T. 2005, A\&A, 433, 101 
Piffaretti, R., \& Kaastra, J. 2006, A\&A, 453, 423

Pizzolato, F., \& Soker, N. 2005, ApJ, 632, 821

Quataert, E. 1998, ApJ, 500, 978

Quataert, E., \& Narayan, R. 2000, ApJ, 528, 236

Rasera, Y., \& Chandran, B. 2007, ApJ, submitted

Rebusco, P., Churazov, E., Bhringer, H., Forman, W. 2005, MNRAS, 359, 1041

Rechester, R., \& Rosenbluth, M. 1978, Phys. Rev. Lett., 40, 38

Reynolds, C. S. 2002, in ASP Conf. Proc., 250, Particles and Fields in Radio Galaxies, ed. R. A. Laing, \& K. M. Blundell (San Fransisco: ASP), 449

Reynolds, C. S., McKernan, B., Fabian, A., Stone, J., \& Vernaleo, J. 2005, MNRAS

Rosner, R., \& Tucker, W. 1989, ApJ, 338761

Ruszkowski, M., \& Begelman, M. 2002, 581, 223

Ruszkowski, M., Bruggen, M., \& Begelman, M. 2004a, ApJ, 611, 158

Ruszkowski, M., Bruggen, M., \& Begelman, M. 2004b, ApJ, 615, 675

Sazonov, S. Y., Ostriker, J. P., Ciotti, L., \& Sunyaev, R. A. 2005, MNRAS, 358, 168

Schekochihin, A. A., Cowley, S. C., Kulsrud, R. M., Hammett, G. W., \& Sharma, P. 2006, ApJ, 629,139

Schekochihin, A. A. \& Cowley, S. C. 2006, Phys. Plasmas, 13, 056501

Schombert, J. M. 1987, APJS, 64, 643

Soker, N. 2006, New Astronomy, 12, 38

Spitzer, L, \& Harm, R. 1953, Phys. Rev., 89, 977

Springel, V., Di Matteo, T., \& Hernquist, L. 2005, MNRAS, 361, 776

Suginohara, T., \& Ostriker, J. 1998, ApJ, 507, 16

Tabor, G., \& Binney, J. 1993, MNRAS, 263, 323

Tan, J., \& Blackman, E. 2005, MNRAS, 362, 983

Taylor, G., Fabian, A., Allen, S. 2002, MNRAS, 334, 769

Taylor, G., Govoni, F., Allen, S., Fabian, A. 2001, MNRAS, 326, 2

Tamura, T. et al 2001, A\&A, 365, L87

Tornatore, L., Borgani, S., Springel, V., Matteucci, F., Menci, N., \& Murante, G. 2003, MNRAS, 342,1025 
Tozzi, P., \& Norman, C. 2001, ApJ, 546, 63

Travis, L., \& Matsushima, S. 1973, ApJ, 180, 975

Ulrich, R. 1976, ApJ, 207, 564

Vogt, C., \& Ensslin, T. 2003, A\&A, 412, 373

Vogt, C., \& Ensslin, T. 2005, A\&A, 434, 67

Voigt, L., \& Fabian, A. 2004, MNRAS, 347, 1130

Werner, N., Kaastra, J. S., Takei, Y., Lieu, R., Vink, J., \& Tamura, T. 2007, A\&A, 468, 849

Worthey, G. 1994, ApJ, 95, 107

Xiong, D. R. 1991, Proc. Astr. Soc. Australia, 9, 26

Yan, H., \& Lazarian, A. 2004, ApJ, 614, 757

Zakamska, N., \& Narayan, R. 2003, ApJ, 582, 162 